\title{
Dynamic Transitions of Epilepsy Waveforms Induced by Astrocyte Dysfunction and Electrical Stimulation
}

\author{
Honghui Zhang $\mathbb{D},{ }^{1}$ Zhuan Shen, ${ }^{1}$ Qiangui Zhao, ${ }^{1}$ Luyao Yan, ${ }^{1}$ Lin Du, ${ }^{1}$ and Zichen Deng $\mathbb{D}^{2}$ \\ ${ }^{1}$ School of Mathematics and Statistics, Northwestern Polytechnical University, Xi'an 710072, China \\ ${ }^{2}$ School of Aeronautics, Northwestern Polytechnical University, Xi'an 710072, China \\ Correspondence should be addressed to Zichen Deng; dweifan@nwpu.edu.cn
}

Received 30 August 2020; Revised 14 October 2020; Accepted 22 October 2020; Published 17 November 2020

Academic Editor: Rubin Wang

Copyright ( 92020 Honghui Zhang et al. This is an open access article distributed under the Creative Commons Attribution License, which permits unrestricted use, distribution, and reproduction in any medium, provided the original work is properly cited.

\begin{abstract}
Experimental studies have shown that astrocytes participate in epilepsy through inducing the release of glutamate. Meanwhile, considering the disinhibition circuit among inhibitory neuronal populations with different time scales and the feedforward inhibition connection from thalamic relay nucleus to cortical inhibitory neuronal population, here, we propose a modified thalamocortical field model to systematically investigate the mechanism of epilepsy. Firstly, our results show that rich firing activities can be induced by astrocyte dysfunction, including high or low saturated state, high- or low-frequency clonic, spikewave discharge (SWD), and tonic. More importantly, with the enhancement of feedforward inhibition connection, SWD and tonic oscillations will disappear. In other words, all these pathological waveforms can be suppressed or eliminated. Then, we explore the control effects after different external stimulations applying to thalamic neuronal population. We find that singlepulse stimulation can not only suppress but also induce pathological firing patterns, such as SWD, tonic, and clonic oscillations. And we further verify that deep brain stimulation can control absence epilepsy by regulating the amplitude and pulse width of stimulation. In addition, based on our modified model, $3: 2$ coordinated reset stimulation strategies with different intensities are compared and a more effective and safer stimulation mode is proposed. Our conclusions are expected to give more theoretical insights into the treatment of epilepsy.
\end{abstract}

\section{Introduction}

Epilepsy has been the second worldwide neurological disorder that affects over 65 million people [1]. As a type of generalized epilepsy, absence epilepsy is featured by periodic 2$4 \mathrm{~Hz}$ SWDs in electroencephalography (EEG) recordings, mainly occurring in children and accompanying a brief impairment of consciousness in seizures [2-4]. Epileptic tonic and clonic seizures are also the primary generalized seizures, seriously affecting the patients' life [5-7]. The EEG of patients with tonic seizures shows high-frequency and lowamplitude rapid discharge activities, while clonic seizures exhibit low-frequency and high-amplitude slow-wave oscillations $[8,9]$. Furthermore, the evolution of tonic to clonic seizures has been observed clinically $[8,9]$. Since the causes of epilepsy are complex and the pathogenesis is still not entirely clear, it has become a major topic for many neuroscientists.
It is traditionally believed that epilepsy mainly results from the imbalance of excitatory and inhibitory factors in the nervous system, such as excitatory or inhibitory synaptic dysfunction and neurotransmitter imbalance [10-12]. Some scholars have pointed out that absence epilepsy was initiated in the cortex [13], some believed that it originated from thalamus $[14,15]$, while others have attributed it to the abnormality of cortical thalamic network [16, 17]. Moreover, many important advances have shown that astrocytes are involved in neuronal firing behaviors [18-21] as well as epilepsy [22-26]. Steinhäuser and Seifert pointed out that the abnormal expression of potassium channels, glutamate receptors, and gap junctions in astrocytes can induce epileptic discharges [22, 23]. Crunelli et al. proposed that astrocytes can provide an important basis for epilepsy treatment [26]. Tian et al. showed that glutamate released by astrocytes stimulates neuronal excitatory receptors, possibly causing 
hyperexcitatory epileptic discharges [27-30]. Li et al. had given insight into the effect of astrocyte $\mathrm{Ca}^{2+}$ oscillation on the generation, spread, and inhibition of epilepsy from the perspective of dynamics [31-33].

Researches based on computational models, including mesoscopic neuron population models [34], mean field models [35, 36], and neural field models $[37,38]$, have greatly promoted people's understanding of epilepsy from different levels. As one of the representatives of the neural field model, Taylor model can perfectly simulate the $2-4 \mathrm{~Hz}$ SWD that characterizes absence seizures $[37,38]$. Recently, scholars have proposed many improved models to study absence epilepsy $[39,40]$. For examples, Fan et al. investigated the combined effects of feedforward inhibition and excitation in thalamocortical circuit on the transition behaviors of epileptic seizures by introducing the feedforward excitation from thalamic relay nucleus to excitatory pyramidal neuronal into the thalamocortical network [39]. Liu et al. constructed another modified thalamocortical network by considering a disinhibition neural population [40].

It was estimated that one-third of epilepsy patients have difficulties getting good treatment with antiepileptic drugs [41]. Surgical resection may be a good treatment choice just for a minority of patients [42]. Finding new treatments is still an urgent problem in the field of antiepileptic research. Taylor et al. found that the transitions between normal and epileptic states can be induced by single-pulse stimulation and further studied the effect of stimulation on SWD [38]. Ge et al. presented a robust closed-loop control of SWD based on a thalamocortical computational model of absence epilepsy [43]. Wang et al. tried different stimulation strategies, including deep brain stimulation (DBS), 1:0 coordinated reset stimulation (CRS), and 3:2 CRS [44]. These methods are all attempts based on theoretical models, but they can give some scientific enlightenments to the direction of clinical treatment.

In this paper, we provide a possibility that astrocytes are involved in epileptic seizure based on a modified neural field model, which will be introduced in detail in the next section. Then, we are dedicated to studying the transition dynamics induced by astrocyte dysfunction and focus on the control effects after different stimulations applying to thalamic neuronal population. Finally, we give the discussions and conclusions.

\section{Materials and Methods}

2.1. Description of the Modified Neural Field Model. Pi et al. proved that there are mutual effects among different inhibitory neurons with different time scales [45]. Beverlin et al. showed that feedforward inhibition from thalamic relay nucleus (TC) to inhibitory interneuron has even more stronger functions on the cortex than the feedforward excitation from TC to excitatory pyramidal neuronal (PY) [46]. To the best of our knowledge, although Fan et al. and Liu et al. had considered the above-mentioned physiological phenomena in their computational models separately $[39,40]$, no scholars have considered these two facts at the same time. Motivated by these, we propose a more comprehensive

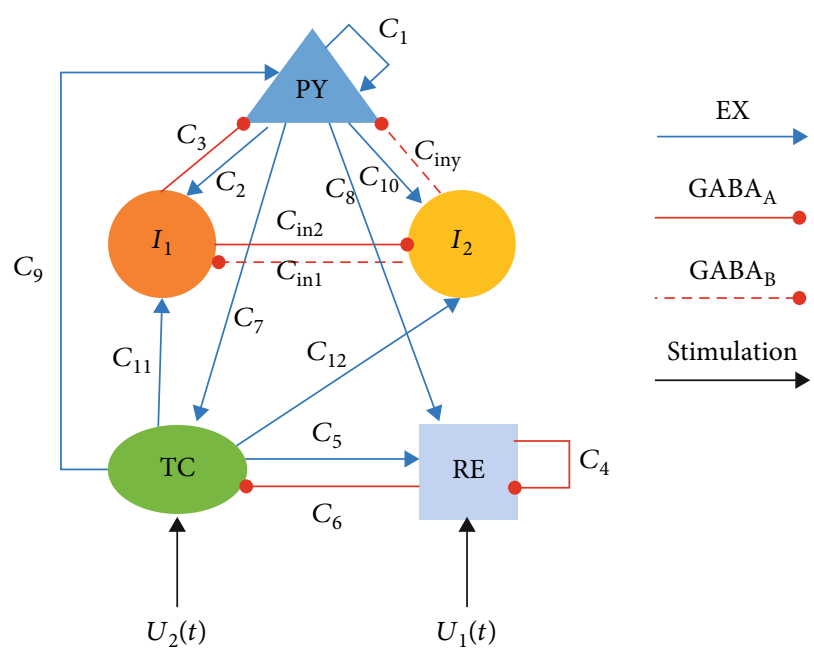

Figure 1: The topological structure of the model, which is composed of excitatory pyramidal neuronal (PY), inhibitory interneuron with different time scales $\left(I_{1}, I_{2}\right)$, thalamic relay nucleus (TC), and thalamic reticular nucleus (RE). The blue lines indicate the excitatory projection of glutamate receptors, while red solid lines and red dashed lines represent the inhibitory projection mediated by $\mathrm{GABA}_{\mathrm{A}}$ and $\mathrm{GABA}_{\mathrm{B}}$, respectively. $U_{1}(t)$ and $U_{2}(t)$ are the stimulus on RE and TC.

model to systematically investigate the effects of important model parameters on the transition behaviors of epileptic seizures including tonic-clonic and absence seizures.

The improved model is composed of cortical subnetwork and thalamus subnetwork. The former is composed of excitatory pyramidal neuronal (PY) and inhibitory interneuron with different time scales $\left(I_{1}, I_{2}\right)$, while the latter is formed from the thalamic relay nucleus (TC) and thalamic reticular nucleus (RE). The excitatory projections of glutamate receptors are represented by blue lines, and the inhibitory projections mediated by $\mathrm{GABA}_{\mathrm{A}}$ and $\mathrm{GABA}_{\mathrm{B}}$ are denoted by red solid lines and red dashed lines, respectively. In addition, the stimulus considered are applied to RE and TC, which are indicated by $U_{1}(t)$ and $U_{2}(t)$, respectively (see Figure 1). Therefore, the nonlinear system can be described as below:

$$
\begin{aligned}
\frac{d \mathrm{PY}}{d t} & =\sigma_{1}\left(h_{\mathrm{PY}}-\mathrm{PY}+C_{1} f(\mathrm{PY})-C_{3} f\left(I_{1}\right)+C_{9} f(\mathrm{TC})-C_{\text {iny }} f\left(I_{2}\right)\right) \\
\frac{d I_{1}}{d t} & =\sigma_{2}\left(h_{I_{1}}-I_{1}+C_{2} f(\mathrm{PY})-C_{\mathrm{in} 1} f\left(I_{2}\right)+C_{11} f(\mathrm{TC})\right) \\
\frac{d I_{2}}{d t} & =\sigma_{3}\left(h_{I_{2}}-I_{2}+C_{10} f(\mathrm{PY})-C_{\mathrm{in} 2} f\left(I_{1}\right)+C_{12} f(\mathrm{TC})\right) \\
\frac{d \mathrm{TC}}{d t} & =\sigma_{4}\left(h_{\mathrm{TC}}-\mathrm{TC}-C_{6} g(\mathrm{RE})+C_{7} f(\mathrm{PY})\right)+U_{2}(t), \\
\frac{d \mathrm{RE}}{d t} & =\sigma_{5}\left(h_{\mathrm{RE}}-\mathrm{RE}-C_{4} g(\mathrm{RE})+C_{5} g(\mathrm{TC})+C_{8} f(\mathrm{PY})\right)+U_{1}(t),
\end{aligned}
$$

where PY, $I_{1}, I_{2}$, TC, and RE denote different types of populations and $\sigma_{1}, \sigma_{2}, \sigma_{3}, \sigma_{4}$, and $\sigma_{5}$ are the time scales, while $h_{\mathrm{PY}}, h_{I_{1}}, h_{I_{2}}, h_{\mathrm{TC}}$, and $h_{\mathrm{RE}}$ are external inputs. $C_{i}$ 
TABLE 1: Standard values of main model parameters.

\begin{tabular}{lccccc}
\hline Symbol & Value & Symbol & Value & Symbol & Value \\
\hline$h_{\mathrm{PY}}$ & -0.3 & $\varepsilon$ & $2.5 e+5$ & $C_{8}$ & 2 \\
$h_{I_{1}}$ & -3.4 & $a$ & 2.8 & $C_{9}$ & 1 \\
$h_{I_{2}}$ & -2 & $b$ & 0.5 & $C_{10}$ & 2 \\
$h_{\mathrm{TC}}$ & -2.5 & $C_{1}$ & 1.8 & $C_{11}$ & 0.1 \\
$h_{\mathrm{RE}}$ & -4.5 & $C_{2}$ & 4 & $C_{12}$ & 0.05 \\
$\sigma_{1}$ & 26 & $C_{3}$ & 1.5 & $C_{\text {in1 }}$ & 0.1 \\
$\sigma_{2}$ & 32.5 & $C_{4}$ & 0.1 & $C_{\text {in2 }}$ & 0.3 \\
$\sigma_{3}$ & 30 & $C_{5}$ & 8 & $C_{\text {iny }}$ & 0.1 \\
$\sigma_{4}$ & 2.6 & $C_{6}$ & 1 & & \\
$\sigma_{5}$ & 2.6 & $C_{7}$ & 2 & & \\
\hline
\end{tabular}

$(i=1,2,3, \cdots, 9)$ are strength of coupling between different neuron populations. $U_{1}(t)$ and $U_{2}(t)$ are the stimulus on RE and TC. $f(\bullet)$ and $g(\bullet)$ are transfer functions $[37,38]$, which can be expressed as follows:

$$
\begin{gathered}
f(x)=\frac{1}{1+\varepsilon^{-x}}, \\
g(y)=a y+b,
\end{gathered}
$$

where $x=\mathrm{PY}, I_{1}, I_{2}$, and $y=\mathrm{TC}$, RE. $\varepsilon$ and $a$ determine the steepness of the two transfer functions, respectively, and $b$ is a constant. In addition, unless noted otherwise, values of model parameters in our simulations are consistent with previous studies [37-40]. New parameters are set within a reasonable range to study the transition behaviors of epileptic seizures (see Table 1).

2.2. Numerical Simulation Methods and Data Analysis. In this paper, the differential equations are solved by the standard fourth-order Runge-Kutta integration method under the MATLAB (MathWorks, USA) simulating environment. And the time window is $30 \mathrm{~s}$ with integration step $1 \mathrm{~ms}$. The time series of each cortical neuron population are averaged and used for data analysis (i.e., $\left.\left(\mathrm{PY}+I_{1}+I_{2}\right) / 3\right)$. The bifurcation diagrams are plotted by calculating the stable local minimum and maximum values of $\left(\mathrm{PY}+I_{1}+I_{2}\right) / 3$ as parameters gradually vary. At the same time, we provide dynamical analysis corresponding to some typical state transitions by using the continuation package, AUTO in XPPAUT (available from http://www.math.pitt.edu/bard/ xpp/xpp.html). In addition, we acquire normalized power spectral density by utilizing the fast Fourier transform and the maximum peak frequency is defined as the dominant frequency of the oscillation.

\section{Results}

3.1. Transition Dynamics Induced by Astrocyte Dysfunction. In the central nervous system, there are many cell types capable of expressing glutamate carriers, such as astrocytes and neurons [47]. Only when glutamate overflows, the neuron carrier can play a role. That is to say, the uptake of glutamate by astrocytes is the main way to clear glutamate in the intercellular space $[47,48]$. The weaker the uptake capacity of astrocytes, the greater the uptake capacity that neurons need to bear, and the stronger the coupling connection. In addition, electrophysiological experiments have shown that epilepsy is caused by abnormal information exchange between the cortex and thalamus circuits $[49,50]$. In our model, it is considered that the cortex subnetwork and the thalamus subnetwork are mainly mediated by glutamate receptors [51]. Inspired by these, in this section, we provide a possibility that astrocytes are involved in epileptic seizure and we are dedicated to studying the transition dynamics induced by astrocyte dysfunction. More precisely, we mainly investigate the effect of parameter $C_{7}$.

Firstly, we set $C_{11}=0.1$, and the remaining parameters are consistent with Table 1 . Rich firing modes can be captured as the bifurcation parameter $C_{7}$ varies (see Figure 2(b)). The more functional the astrocytes are, the less glutamate the neurons absorb; that is, the smaller $C_{7}$ is, then the brain will exhibit tonic oscillation (i.e., IV). If astrocytes function normally, the system will show normal low saturated state (i.e., II). However, when the function of astrocytes is inhibited or damaged, the glutamate absorbed by neurons exceed the normal value, and the system shows SWD oscillation (i.e., III). With the further increase in absorption, the system transitions from SWD to low-frequency clonic oscillation (i.e., V). If glutamate accumulates in a large amount, neurons will be overexcited, and the system will transit to high saturation state (i.e., I). The oscillation dominant frequencies of all discharge modes can be observed in the figure's lower panel (see Figure 2(b)) and the time series of different firing modes are shown in Figure 3.

Secondly, without loss of generality, we display the dynamical analysis corresponding to the state transitions in Figure 2(b) (see Figure 5). For smaller $C_{7}$ (i.e., $C_{7}<0.24$ ), there is a monostable region consisting of one unstable fixed point and one stable limit cycle; hence, all the simulations converge to the stable limit cycle, and system exhibits tonic oscillations. Then, the tonic oscillation disappears and the low saturated state appears following a supercritical Hopf bifurcation (i.e., $\mathrm{HB}_{1}$ ). Next, with the further increase of $C_{7}$, the system successively undergoes the fold of cycle bifurcation $\left(\mathrm{LPC}_{1}\right)$ and the subcritical Hopf bifurcation $\left(\mathrm{HB}_{2}\right)$ and evolves into a bistable region (i.e., $1.69 \leq \mathrm{C}_{7}$ $\leq 2.16$ ). When $2.16 \leq C_{7} \leq 3.47$, the system is in monostable state until the subcritical Hopf bifurcation $\left(\mathrm{HB}_{3}\right)$ appears. Immediately afterwards, the system transits to the second bistable area (i.e., $3.47<C_{7} \leq 5.01$ ). Last, all the simulations converge to the steady high saturated state due to the fourth fold limit cycle bifurcation $\left(\mathrm{LPC}_{4}\right)$. In addition, it is worth noting that during our simulations, the initial values are set to $[0,0$, $0,0,0]$. In the above two bistable regions, the initial values are close to the side of separatrix near stable limit cycle, so the system will converge to the periodic oscillation (see Figure 2(b)).

Thirdly, considering the complexity of neural networks, we give the dynamic transition phenomenon induced by $C_{7}$ when $C_{11}$ is set to 0.02 (see Figure 2(a)), 0.5 (see Figure 2(c)), and 1 (see Figure 2(d)), respectively. If $C_{11}=$ 0.02 , tonic oscillation will disappear. For the larger $C_{11}$, the 

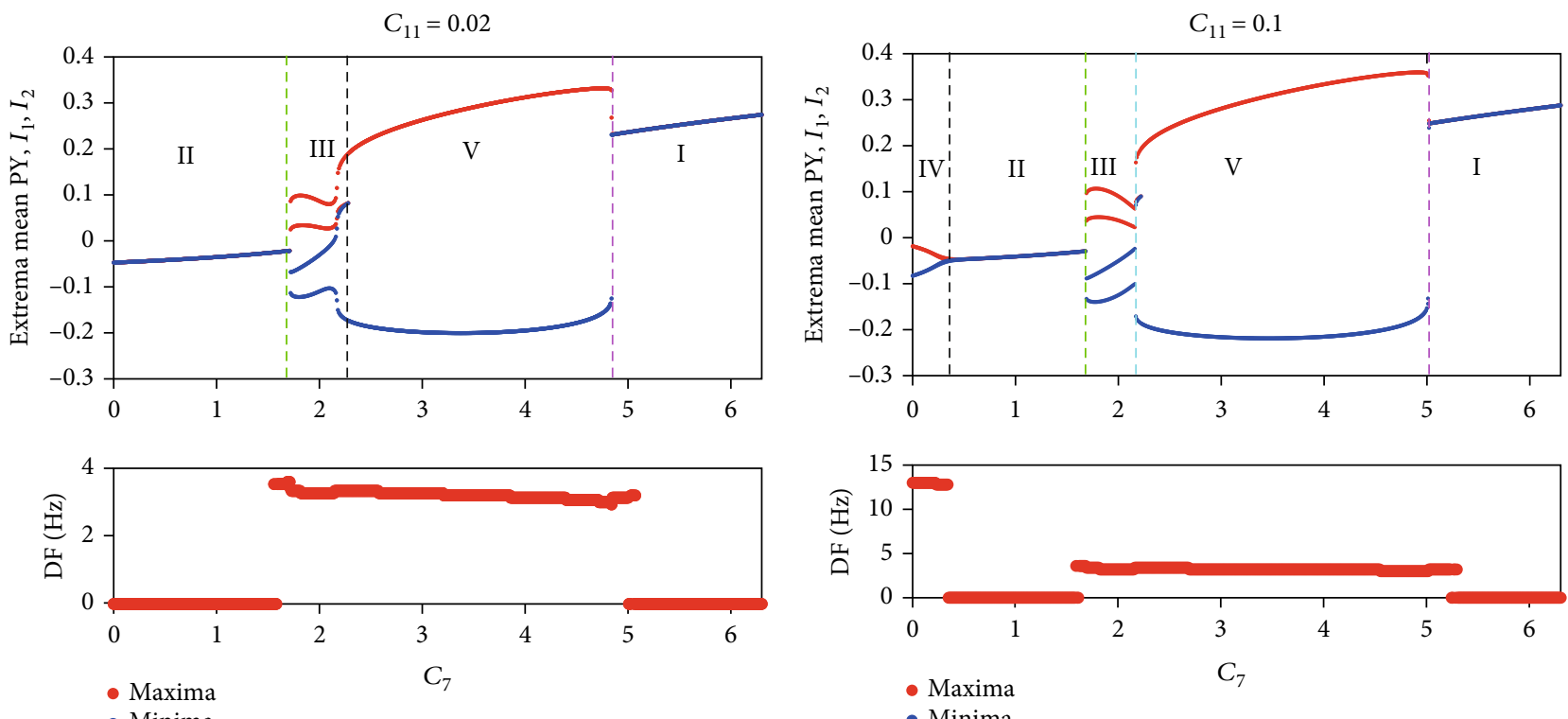

(a)
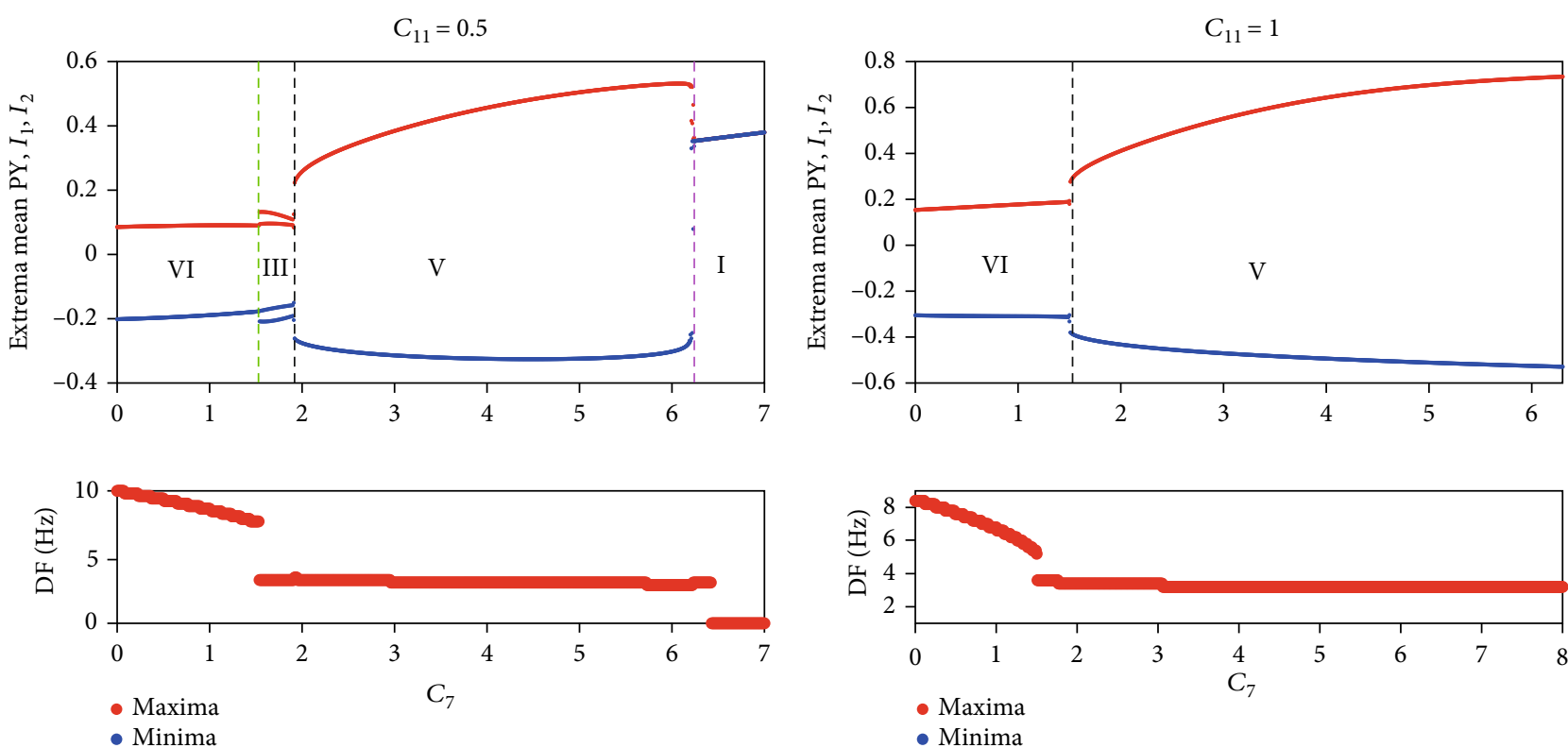

(c)

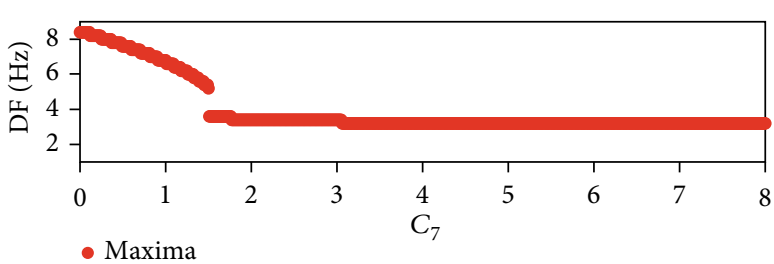

- Minima

(d)

FIgURE 2: The bifurcation diagram (upper panels) and their corresponding dominant frequencies (lower panels) induced by $C_{7}$, when $C_{11}$ is set to $0.02(\mathrm{a}), 0.1$ (b), 0.5 (c), and 1 (d), respectively. Six firing modes can be observed such as I (high saturated state), II (low saturated state), III (SWD), IV (tonic), V (low-frequency clonic), and VI (high-frequency clonic). The dominant frequencies of states I and II are $0 \mathrm{~Hz}$, of states III and V are between $2 \mathrm{~Hz}$ and $4 \mathrm{~Hz}$, and of state VI are between $5 \mathrm{~Hz}$ and $10 \mathrm{~Hz}$, and the dominant frequencies of state IV are up to $13 \mathrm{~Hz}$.

system can exhibit high-frequency clonic (i.e., VI), whose dominant frequency is between $5 \mathrm{~Hz}$ and $10 \mathrm{~Hz}$ (see Figure 2(c)). However, only high-frequency clonic and lowfrequency clonic can be observed in the inspection window when $C_{11}=1$.

Lastly, in order to observe the interaction between $C_{7}$ and $C_{11}$ generally, we show the state type and dominant frequency when $C_{7}$ and $C_{11}$ both change (see Figure 4 ). Rich firing states can be seen by us, such as I (high saturated state), II (low saturated state), III (SWD), IV (tonic), V (low-frequency clonic), and VI (high-frequency clonic). With the enhancement of feedforward inhibition connection, SWD, tonic, and saturated state will disappear. In addition, the dominant frequencies of states I and II are $0 \mathrm{~Hz}$, states III and $\mathrm{V}$ are between $2 \mathrm{~Hz}$ and $4 \mathrm{~Hz}$, and state VI are between $5 \mathrm{~Hz}$ and $10 \mathrm{~Hz}$, while the dominant frequencies of state IV are greater than $10 \mathrm{~Hz}$. Perhaps, epilepsy can be controlled by regulating the uptake of glutamate by astrocytes. 


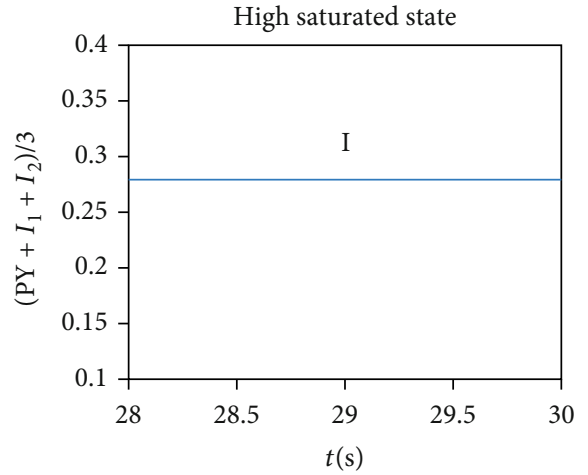

(a)

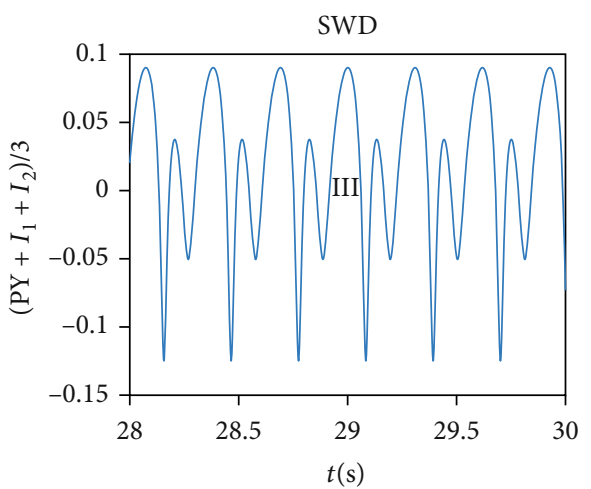

(c)

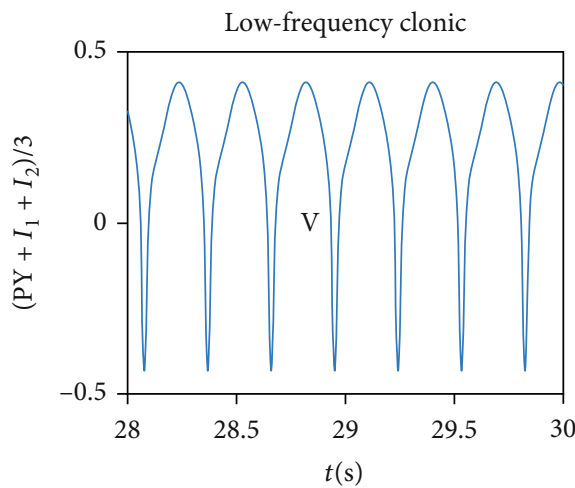

(e)

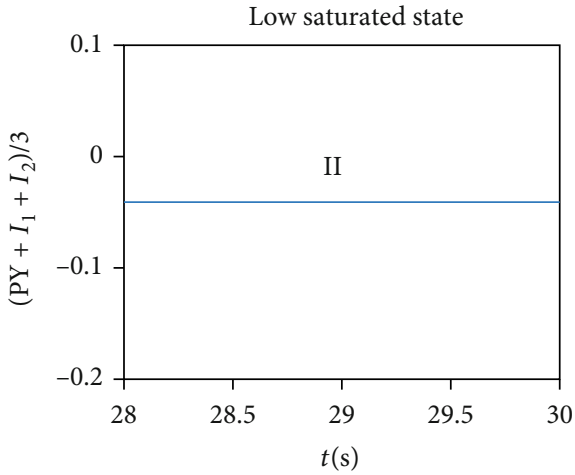

(b)

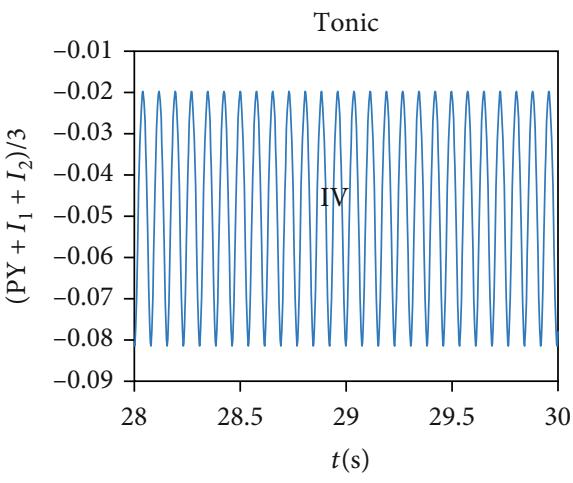

(d)

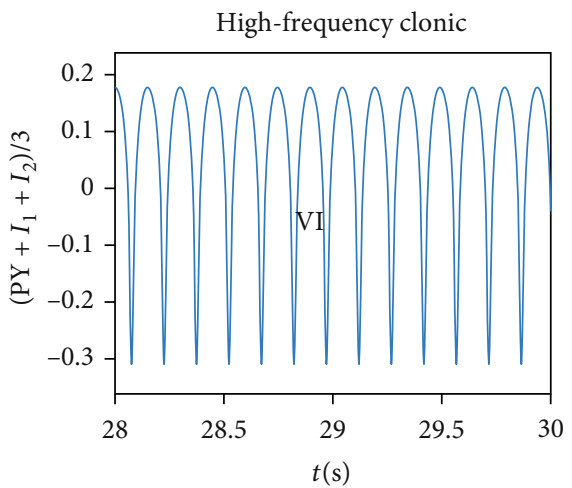

(f)

Figure 3: The time series of different firing modes in Figures 2 and 4: (a) high saturated state, $\left(C_{7}, C_{11}\right)=(6,0.1)$; (b) low saturated state, $\left(C_{7}, C_{11}\right)=(1,0.1)$; (c) SWD, $\left(C_{7}, C_{11}\right)=(2,0.1)$; (d) tonic, $\left(C_{7}, C_{11}\right)=(0.02,0.1)$; (e) low-frequency clonic, $\left(C_{7}, C_{11}\right)=(2,1)$; $(\mathrm{f})$ highfrequency clonic, $\left(C_{7}, C_{11}\right)=(1,1)$.

3.2. Control of Single-Pulse Stimulation. As a gate controller of the thalamus and an important part of the cortexthalamic circuit, RE plays an extremely important role in the activation of absence seizures [52, 53]. It has been reported that $\mathrm{RE}$ is involved in tonic seizures and clonic seizures [54, 55], and different types of seizures correspond to different levels of RE's excitability. In addition, single-pulse stimulation has been suggested to prematurely terminate the seizure $[18,56]$. Hence, in the following sections, we focus on the dynamic transitions after single-pulse stimulation acting on RE.

Firstly, we care the effects of single-pulse stimulation when the system is in pathological states. Obviously, rich dynamic transitions can be captured after single-pulse stimulation acting on $\mathrm{RE}$ at $t=10 \mathrm{~s}$ when the system is in SWD originally (see Figure 6(a)). It is clear that low-frequency clonic and high saturated state can be induced by applying a negative stimulus inputs to RE. On the contrary, when stimulus inputs are positive, SWD, low saturated state, and tonic appear successively as the strength of stimulation increases. The corresponding dominant frequencies and the specific time series also are plotted in Figures 6(a) and 6(b). If we make the initial states to be tonic, we can easily see that tonic oscillation can transit to high saturated state, lowfrequency clonic, low saturated state, and even tonic (see Figures $6(\mathrm{c})$ and $6(\mathrm{~d}))$. Furthermore, when the initial state 


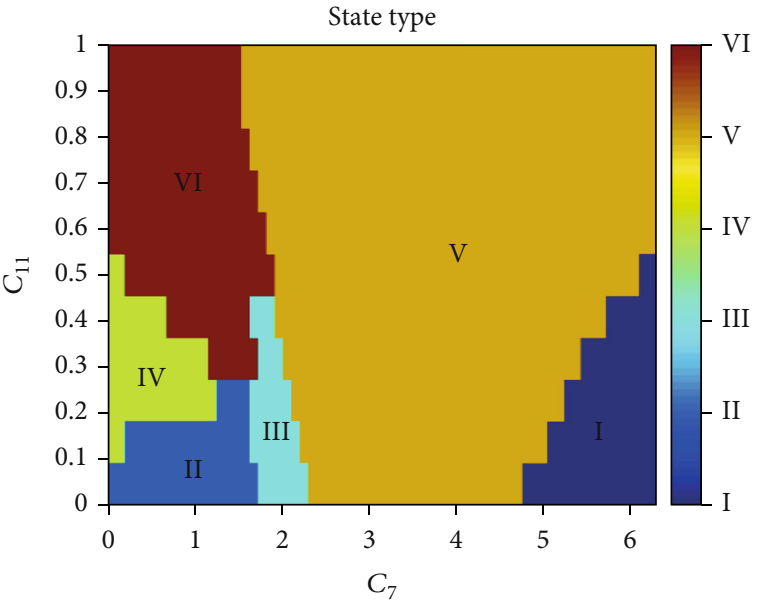

(a)

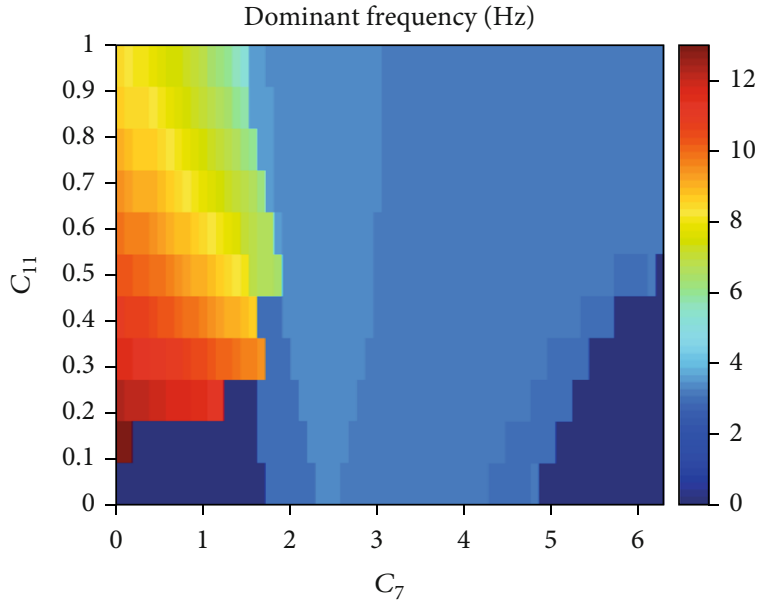

(b)

Figure 4: Different firing states (a) and variations of corresponding dominant frequencies (b) are shown on the parameter plane $\left(C_{7}, C_{11}\right)$, where we can observe rich firing states such as I (high saturated state), II (low saturated state), III (SWD), IV (tonic), V (low-frequency clonic), and VI (high-frequency clonic). In addition, the dominant frequencies of states I and II are $0 \mathrm{~Hz}$, of states III and V are between $2 \mathrm{~Hz}$ and $4 \mathrm{~Hz}$, and of state VI are between $5 \mathrm{~Hz}$ and $10 \mathrm{~Hz}$, while the dominant frequencies of state IV are greater than $10 \mathrm{~Hz}$.

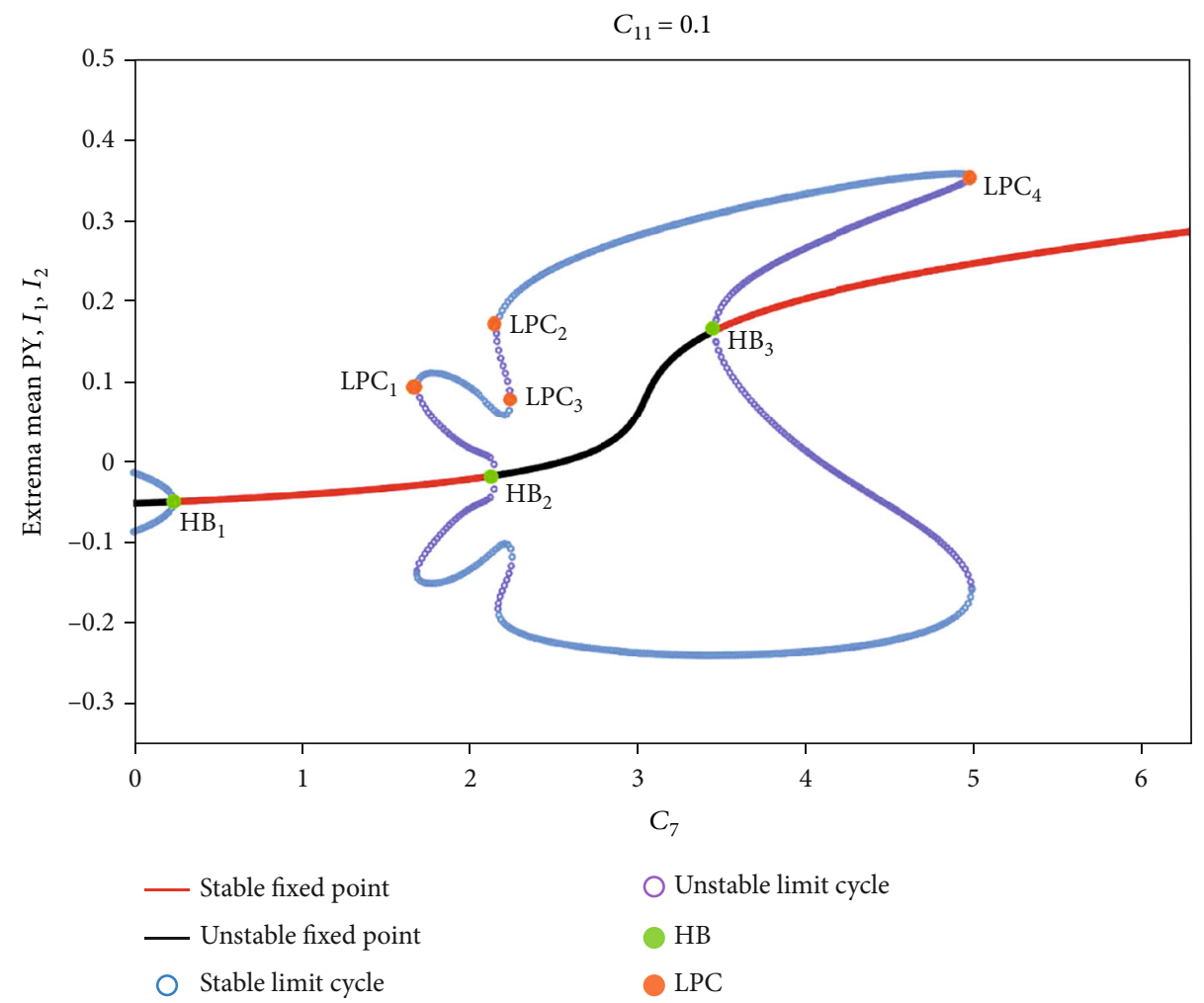

Figure 5: Dynamical analysis corresponding to the state transitions in Figure 2(b). As $C_{7}$ increases, the system successively undergoes the supercritical Hopf bifurcation $\left(\mathrm{HB}_{1}\right)$, the fold of cycle bifurcation $\left(\mathrm{LPC}_{1}\right)$, the subcritical Hopf bifurcation $\left(\mathrm{HB}_{2}\right), \mathrm{LPC}_{2}, \mathrm{LPC}_{3}$, the subcritical Hopf bifurcation $\left(\mathrm{HB}_{3}\right)$, and $\mathrm{LPC}_{4}$.

is low-frequency clonic, the phenomenon is more fascinating. Similar to the case where the initial state is SWD, five firing modes can be induced (see Figures 6(e) and 6(f)). The specific time series diagram for each transition is shown as an example in Figures 6(b), 6(d), and 6(f), and the corre- sponding strength of stimulation is set to $-2,0.5,2,3,5$, respectively (see Figure 6(f)).

For more details, we also display the dynamical analysis corresponding to the state transitions in Figure 6(e) (see Figure 7). For the much small $A_{\text {sps }}$ (i.e., $A_{\text {sps }}<-1.22$ ), there 

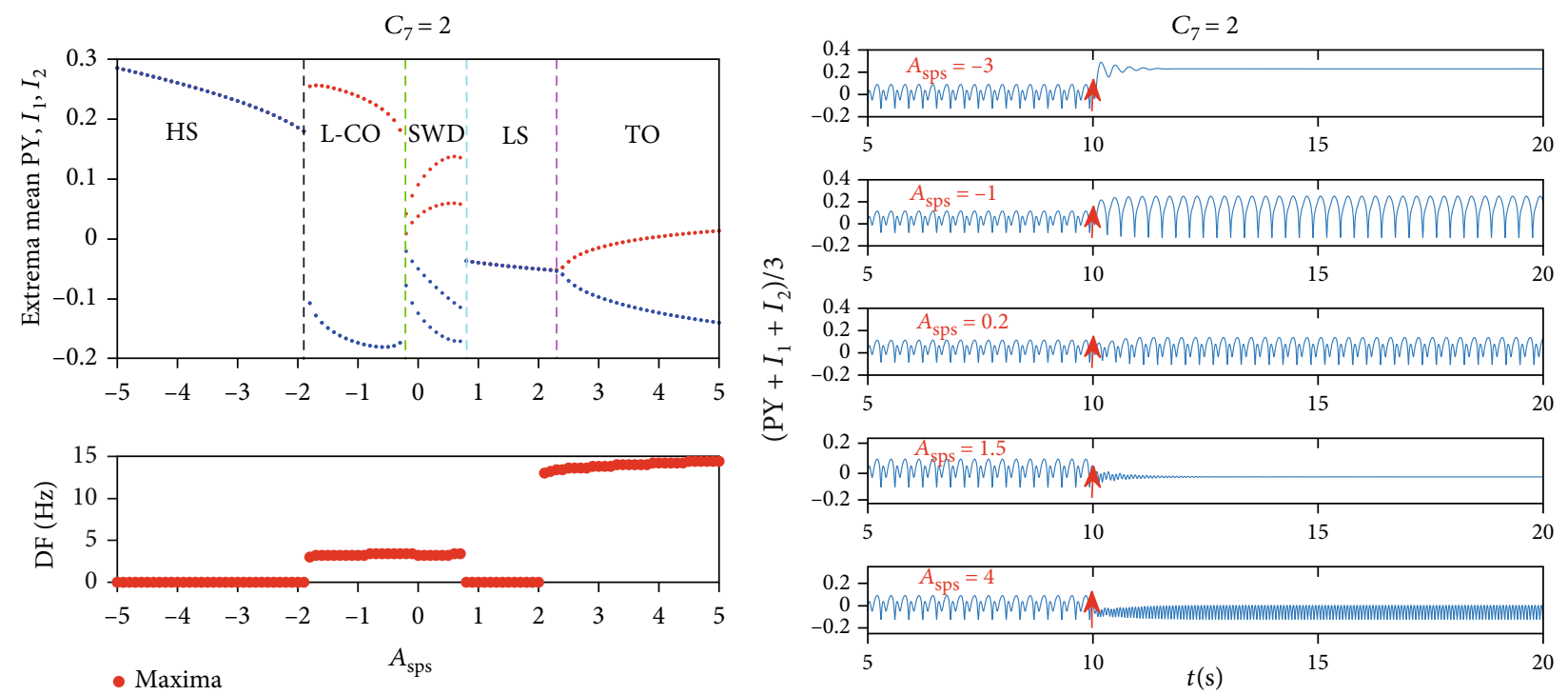

(a)
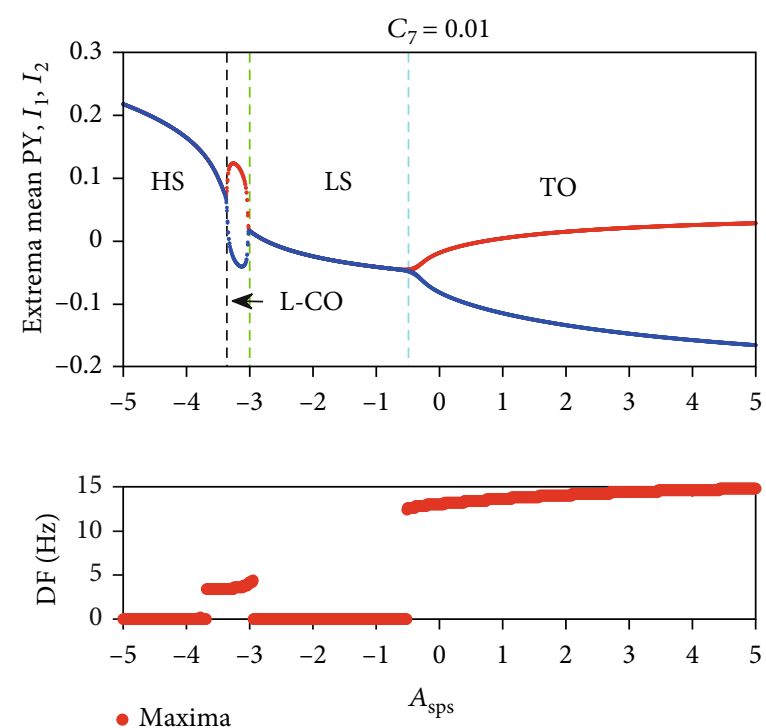

- Maxima $A_{\text {sps }}$

- Minima

(c)

(b)

$C_{7}=0.01$
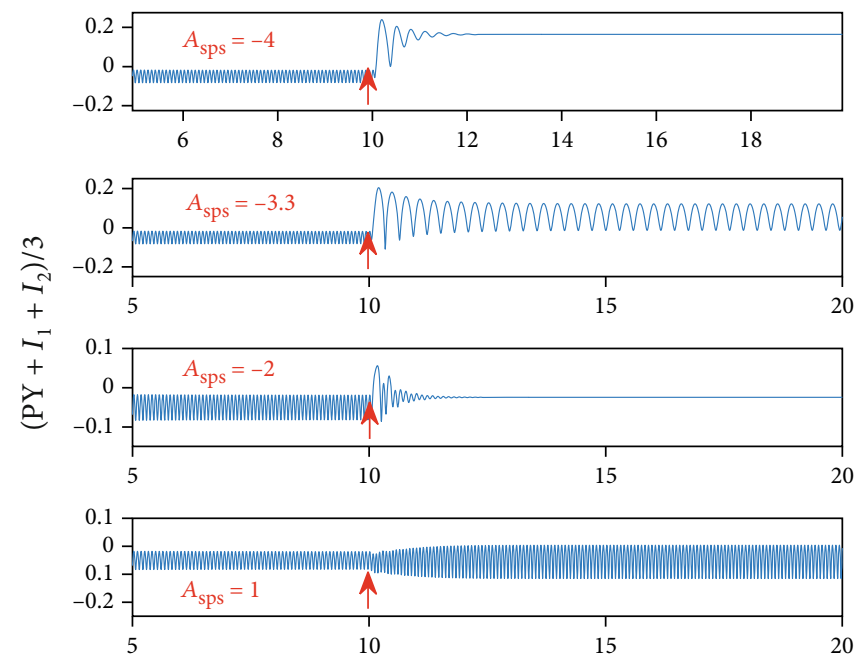

$t(\mathrm{~s})$

(d)

Figure 6: Continued. 

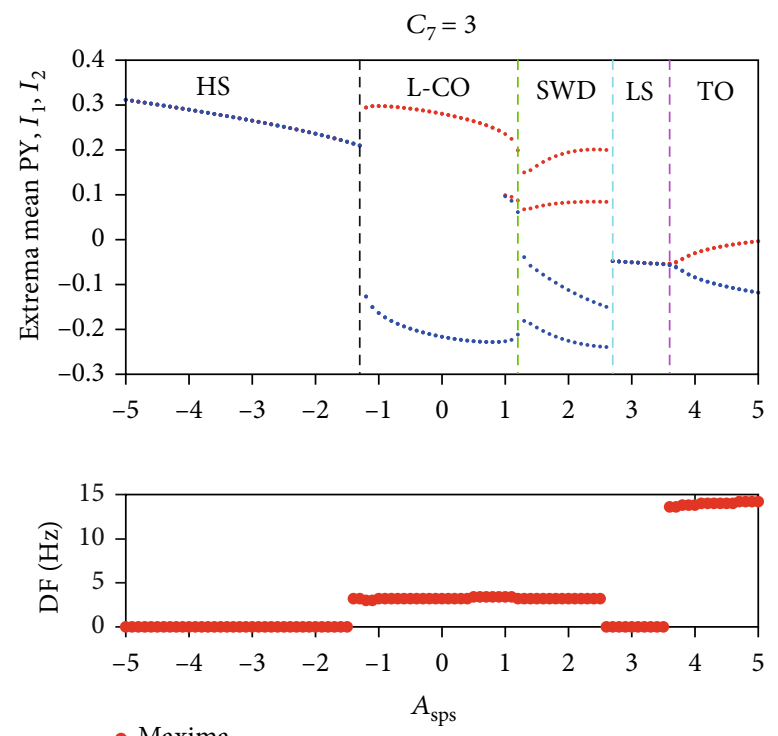

- Maxima
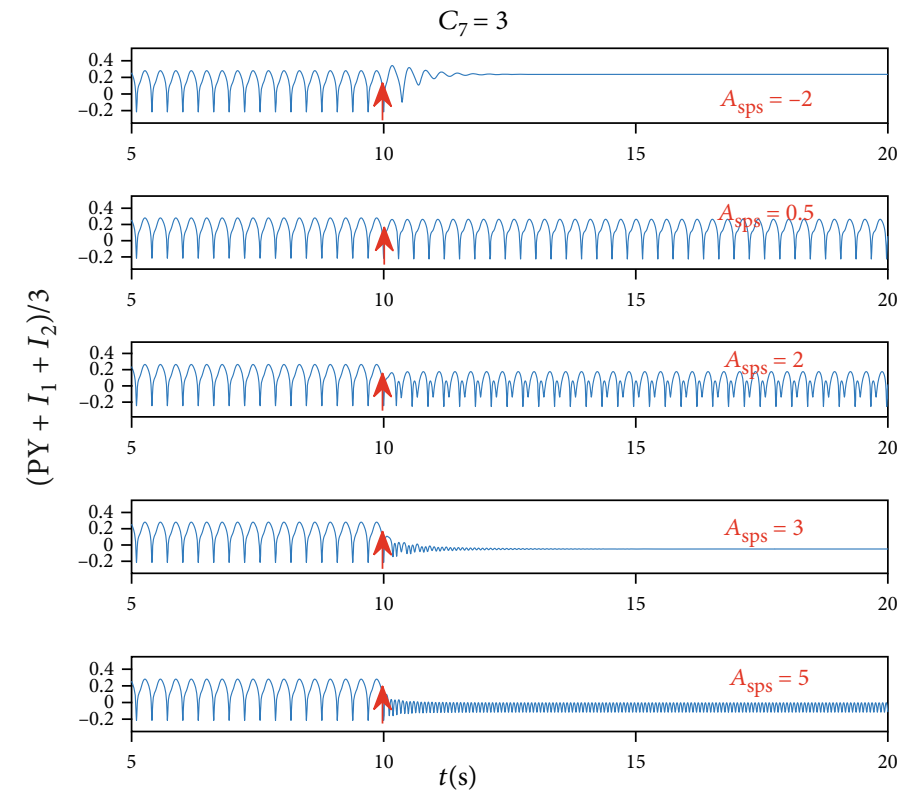

(e)

(f)

Figure 6: The bifurcation diagram (upper panel of (a), (c), and (e)), their corresponding dominant frequencies (lower panel of (a), (c), (e)), and the specific time series (b, d, f) after single-pulse stimulation acting on RE at $t=10 \mathrm{~s}$ when the system is in SWD (a, b), tonic (c, d), and low frequency clonic (e, f) originally. For clarity, TO (tonic), SWD, LS (low saturated state), HS (high saturated state), l-CO (low-frequency clonic oscillations), and the strength of stimulation $\left(A_{\mathrm{sps}}\right)$ are indicated by text labels in each figure.

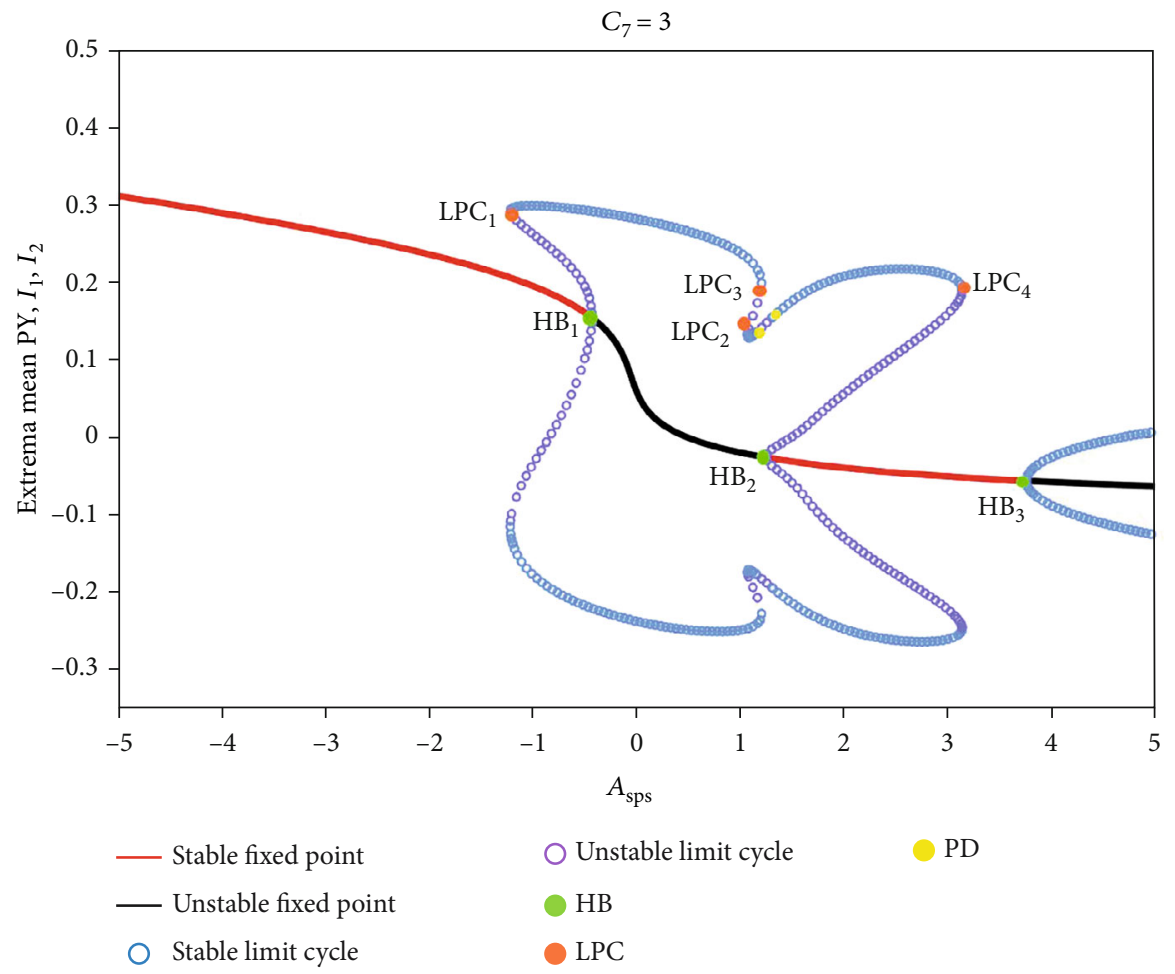

Figure 7: Dynamical analysis corresponding to the state transitions in Figure 7(e). As $A_{\text {sps }}$ increases, the system successively undergoes the fold of cycle bifurcation $\left(\mathrm{LPC}_{1}\right)$, the subcritical Hopf bifurcation $\left(\mathrm{HB}_{1}\right), \mathrm{LPC}_{2}, \mathrm{LPC}_{3}$, period doubling bifurcation $\left(\mathrm{PD}_{1}\right)$, the subcritical Hopf bifurcation $\left(\mathrm{HB}_{2}\right), \mathrm{PD}_{2}, \mathrm{LPC}_{4}$, and the supercritical Hopf bifurcation $\left(\mathrm{HB}_{3}\right)$. 

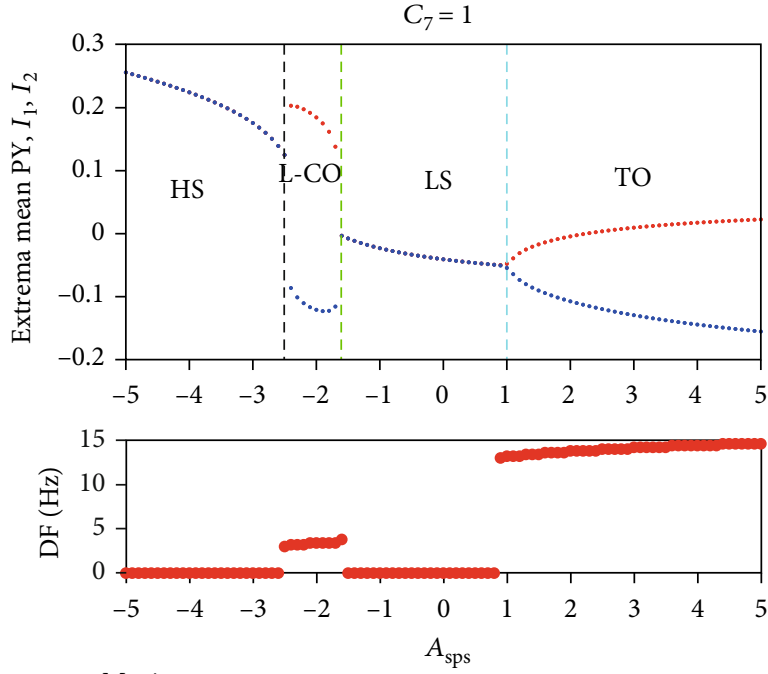

- Maxima

- Minima

(a)
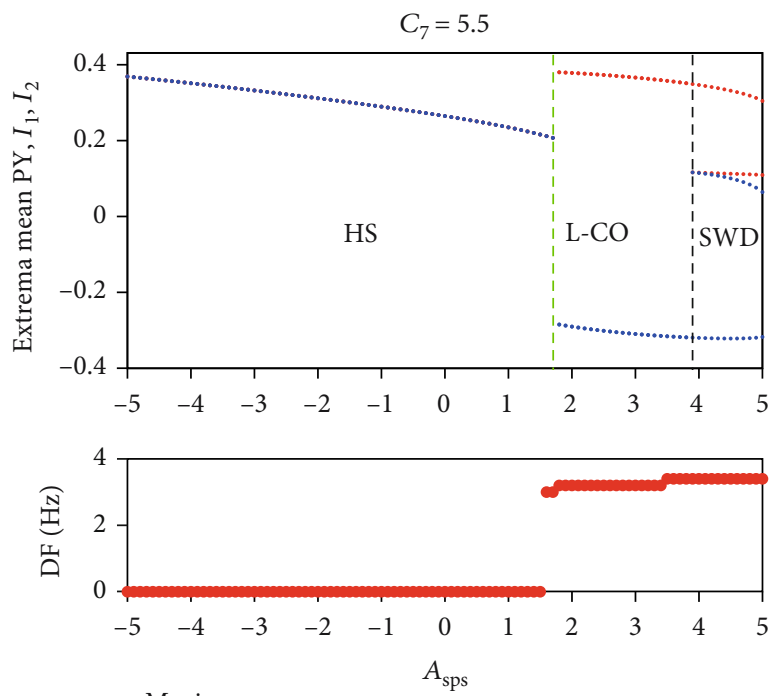

- Maxima

- Minima
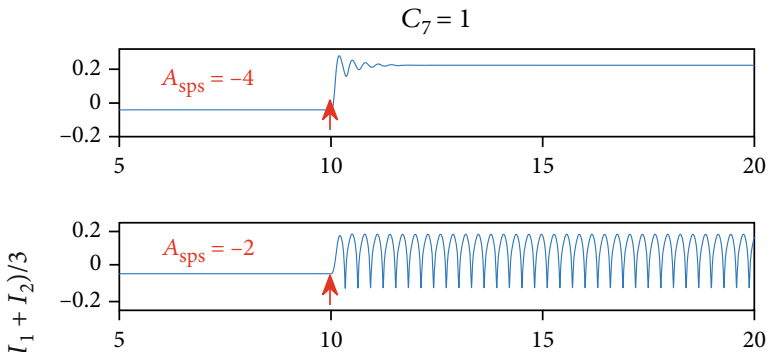

气
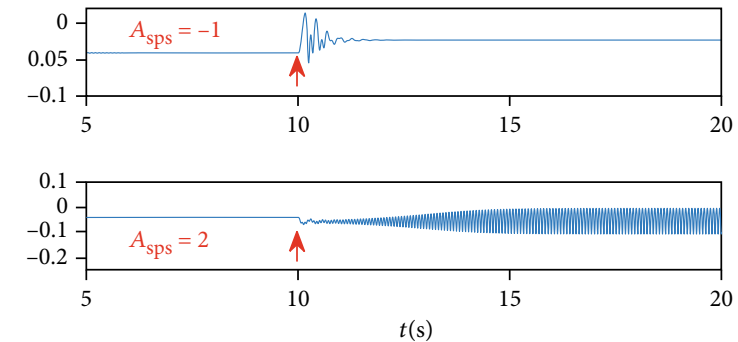

(b)
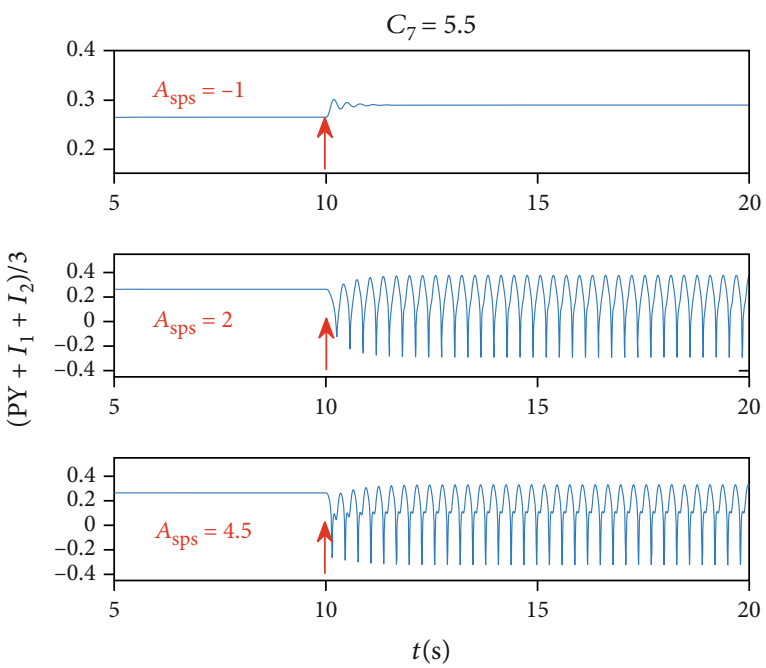

(d)

FIgURE 8: The bifurcation diagram (upper panel of $(a, c)$ ), their corresponding dominant frequencies (lower panel of (a, c)), and the specific time series (b, d) after single-pulse stimulation acting on RE at $t=10 \mathrm{~s}$ when the system is in low saturated state (a, b) and high saturated state (c, d) originally. Similarly, HS (high saturated state), 1-CO (low-frequency clonic oscillations), LS (low saturated state), TO (tonic), SWD, and the strength of stimulation $\left(A_{\mathrm{sps}}\right)$ are indicated by text labels in each figure.

is a monostable region consisting of the stable fixed points, the system converges to stable high saturated state. For the less small $A_{\text {sps }}$ (i.e., $-1.22 \leq A_{\text {sps }}<-0.44$ ), the system transits to the first bistable region between the fold of cycle bifurcation $\left(\mathrm{LPC}_{1}\right)$ and the subcritical Hopf bifurcation $\left(\mathrm{HB}_{1}\right)$. Then, the system successively undergoes $\mathrm{LPC}_{2}, \mathrm{LPC}_{3}$, period doubling bifurcation $\left(\mathrm{PD}_{1}\right)$, and the subcritical Hopf bifurcation $\left(\mathrm{HB}_{2}\right)$ as $A_{\text {sps }}$ increases. At the same time, the system is always in a monostable region (i.e., $-0.44 \leq A_{\text {sps }}<1.20$ ). Next, the second bistable area appears, which is between the subcritical Hopf bifurcation $\left(\mathrm{HB}_{2}\right)$ and $\mathrm{LPC}_{4}$. And the system transits to a monostable region following $\mathrm{LPC}_{4}$. Last, all the simulations converge to the tonic oscillations due to the supercritical Hopf bifurcation $\left(\mathrm{HB}_{3}\right)$. Similarly, the initial values are close to the side of separatrix near stable limit cycle in the above two bistable regions, and the system exhibits the periodic oscillations (see Figure 6(e)).

Secondly, in a similar way, we focus on the dynamical transitions when the system is in stable saturated states. We can see that single-pulse stimulation can not only suppress pathological firing patterns but also induce them. Low saturated state can transit to high saturated state, low-frequency clonic, low saturated state, and tonic oscillation after singlepulse stimulation acting on $\mathrm{RE}$ at $t=10 \mathrm{~s}$, while high 


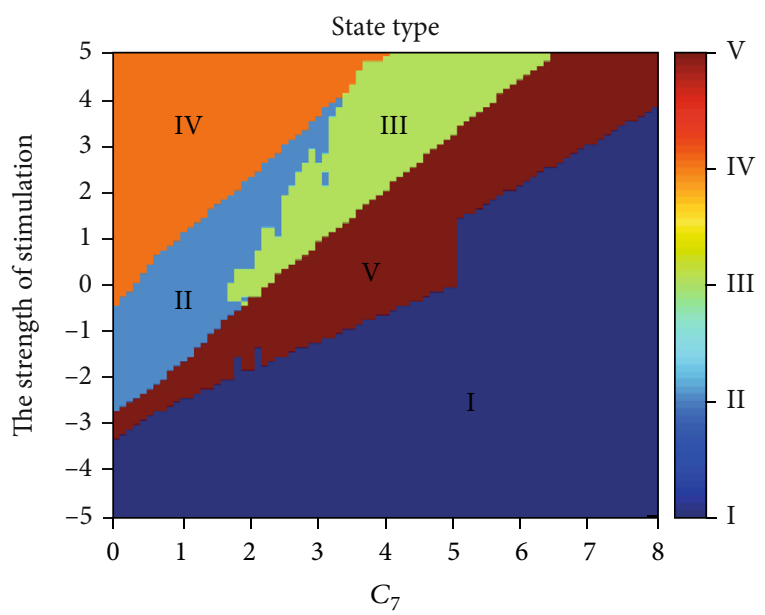

(a)

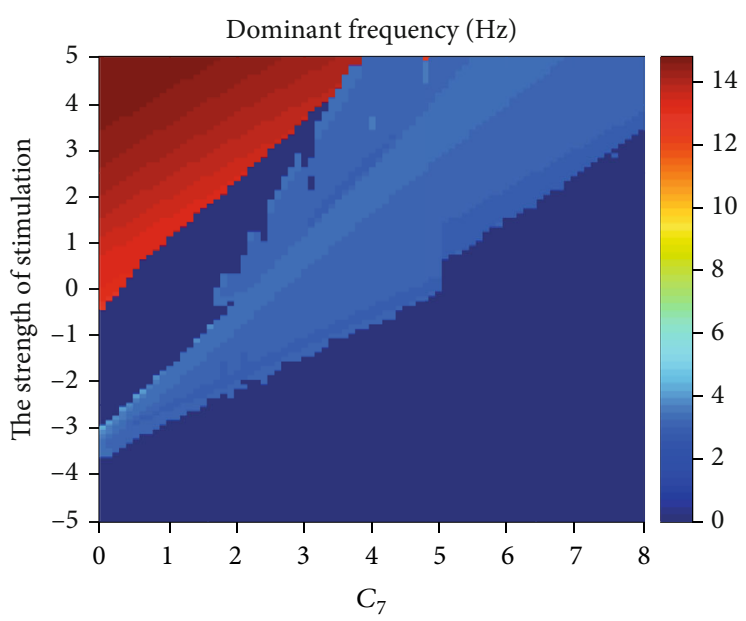

(b)

Figure 9: The state types (a) and dominant frequencies (b) after single-pulse stimulation acting on RE at $t=10 \mathrm{~s}$ when the system is in different initial states. Rich firing states such as I (high saturated state), II (low saturated state), III (SWD), IV (tonic), and V (lowfrequency clonic) can be observed. The dominant frequencies of states I and II are $0 \mathrm{~Hz}$ and of states III and V are between $2 \mathrm{~Hz}$ and $4 \mathrm{~Hz}$, while the dominant frequencies of state IV are nearly $14 \mathrm{~Hz}$.

saturated state can only transit to itself, low-frequency clonic, and SWD (see Figure 8).

Finally, in order to systematically investigate how initial state and stimulus intensity affect state transitions, different firing states (see Figure 9(a)) and variations of corresponding dominant frequency (see Figure 9(b)) after single-pulse stimulation acting on RE are shown on the parameter plane $\left(C_{7}\right.$, the strength of stimulation). There are five different regions representing various effects of stimulation with the different initial states, which are denoted as I (high saturated state), II (low saturated state), III (SWD), IV (tonic), and V (lowfrequency clonic). Specifically, if $C_{7} \leq 1.7$, states I, V, II, and IV appear in succession as the strength of stimulation increases. For larger $C_{7}$ (i.e., $1.7<C_{7} \leq 3.3$ ), the system can display state III. In addition, states II and IV will disappear one after another when $C_{7}$ is around 3.3 and 4, respectively. At last, when $C_{7} \geq 6.5$, we can only observe two states, I and $\mathrm{V}$.

3.3. Control of Deep Brain Stimulation. As one of the effective treatments for Parkinson's disease and epilepsy, deep brain stimulation (DBS) is more and more popular among scholars [57-59]. In this section, we focus on the effect of DBS control strategy on absence epilepsy. Similarly, stimulation is applied to RE, and we employ the typical period step function to simulate DBS as follows:

$$
u(t)=A_{\mathrm{DBS}} \times H(\sin (2 \pi t / p)) \times\left(1-H\left(\sin \left(\frac{2 \pi(t+\delta)}{p}\right)\right)\right)
$$

where $H$ is the Heaviside bivalue step function and $A_{\mathrm{DBS}}, p$, and $\delta$ represent the amplitude, period, and pulse width of stimulus, respectively. Here, we set $p=0.1$.
It is clear that absence epilepsy can be excellently controlled by DBS. On the parameter plane $\left(A_{\mathrm{DBS}}, \delta\right)$, five different firing patterns can be found, which are denoted by I (high saturated state), II (low saturated state), III (SWD), IV (tonic), and V (low-frequency clonic). To be specific, if we apply the positive DBS stimulus to RE at $t=10 \mathrm{~s}$, then the initial state (i.e., SWD) can be firstly controlled to the low saturated state, and then, the system presents tonic oscillation as $A_{\mathrm{DBS}}$ and $\delta$ increase (see Figure 10). However, if DBS stimulus is negative, the system will transit from SWD to low-frequency clonic, then to high saturated state. Certainly, it is worthy to release absence seizure and stimulation side effect as few as possible and taking parameters from region II in Figure 10. In addition, we also specifically show the time series diagram of each area in Figure 10 (see Figure 11). We can clearly see five different transition behaviors, such as SWD to low-frequency clonic, SWD to high saturated state, SWD to SWD, SWD to low saturated state, and SWD to tonic (see Figure 11).

3.4. Control of Coordinated Reset Stimulation. Considering the side effect of single-pulse stimulation and DBS, here, we further explore the control effect of $3: 2$ CRS strategy on absence epilepsy, which is more effective and safer. The stimulus form is similar to the previous research [44], and the stimulation targets are TC and RE. In addition, in order to compare the effect of different stimulation strategies, we introduce two qualitative measurable indexes firstly. One is the percentage reduction (i.e., $\eta$ ) in absence seizures area, which can be described as below:

$$
\eta=\frac{N_{1}-N_{2}}{N_{1}},
$$

where $N_{1}$ and $N_{2}$ denote the mesh numbers occupied by SWD in the two-dimensional parametric plane $\left(C_{7}, C_{11}\right)$ before and after applying CRS to stimulation targets. 


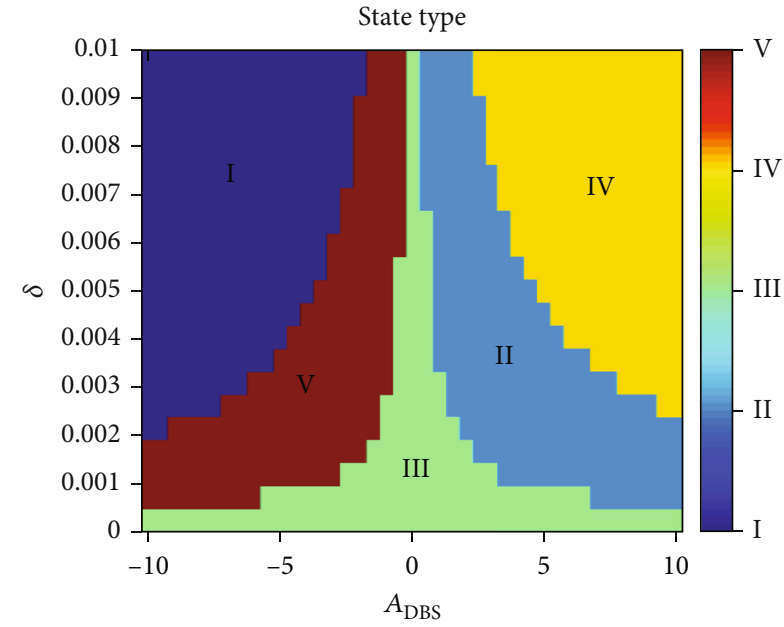

(a)

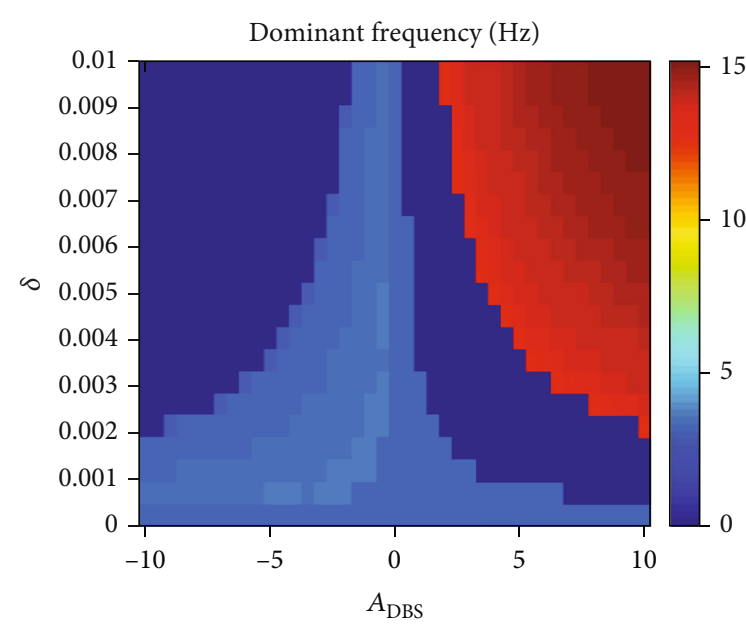

(b)

FIGURE 10: The state types (a) and dominant frequencies (b) after DBS acting on RE at $t=10 \mathrm{~s}$ when the system is in SWD originally. I (high saturated state), II (low saturated state), III (SWD), IV (tonic), and V (low-frequency clonic) can be observed. The dominant frequencies of states I and II are $0 \mathrm{~Hz}$ and of state III and V are between $2 \mathrm{~Hz}$ and $4 \mathrm{~Hz}$, while the dominant frequencies of state IV are between $13 \mathrm{~Hz}$ and $15 \mathrm{~Hz}$.

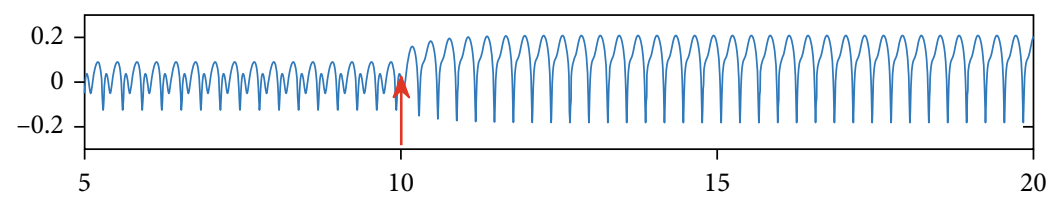

(a)
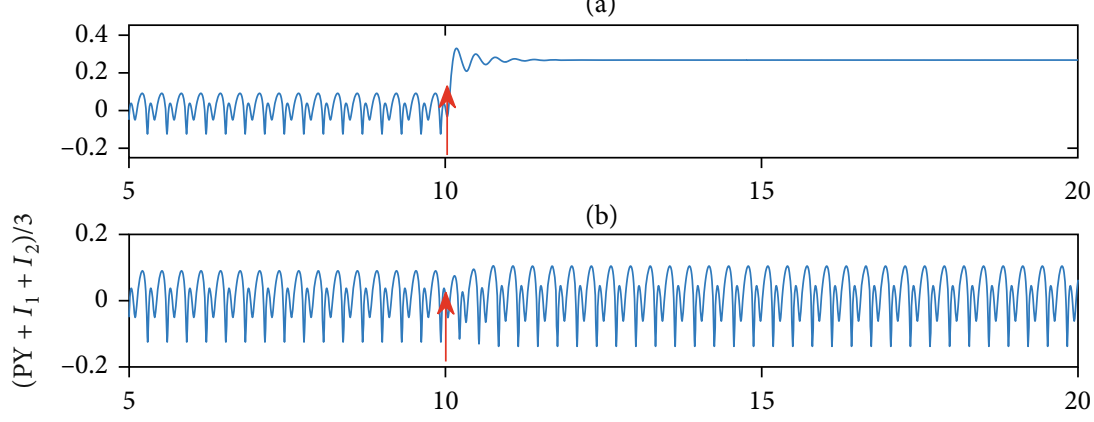

(c)

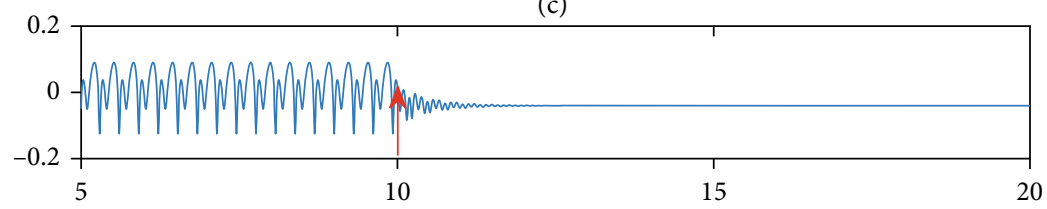

(d)

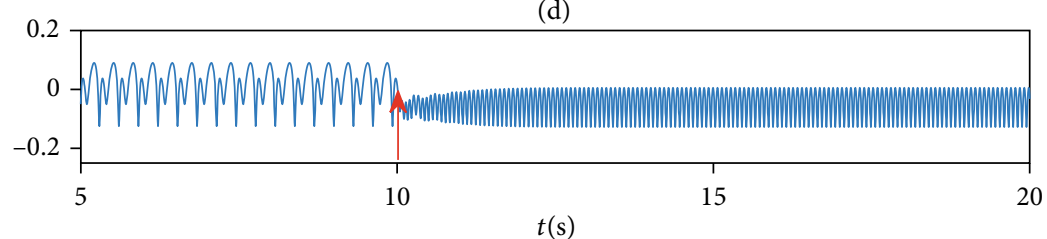

(e)

Figure 11: Transition behaviors under DBS acting on RE at $t=10 \mathrm{~s}$, given by red arrows: (a) SWD to low-frequency clonic, $\mathrm{A}_{\mathrm{DBS}}=-5$, $\delta=0.001$; (b) SWD to high saturated state, $A_{\mathrm{DBS}}=-7, \delta=0.006$; (c) SWD to SWD, $A_{\mathrm{DBS}}=1, \delta=0.001$; (d) SWD to low saturated state, $\mathrm{A}_{\mathrm{DBS}}=5, \delta=0.002$; (e) SWD to tonic, $A_{\mathrm{DBS}}=7, \delta=0.006$. SWD can be controlled into normal or other pathological discharge behaviors by regulating the amplitude and pulse width of stimulation. 


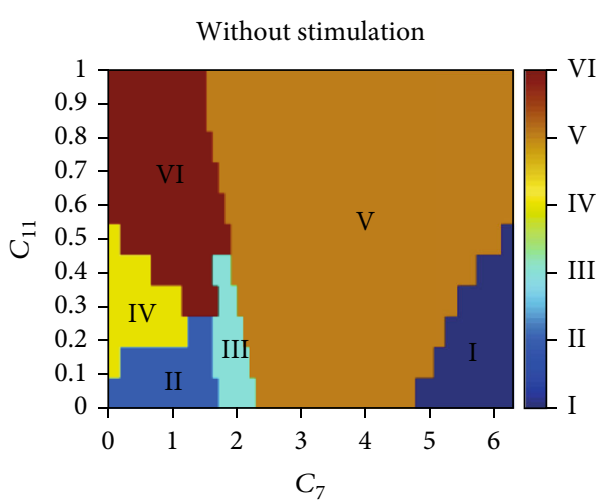

(a)

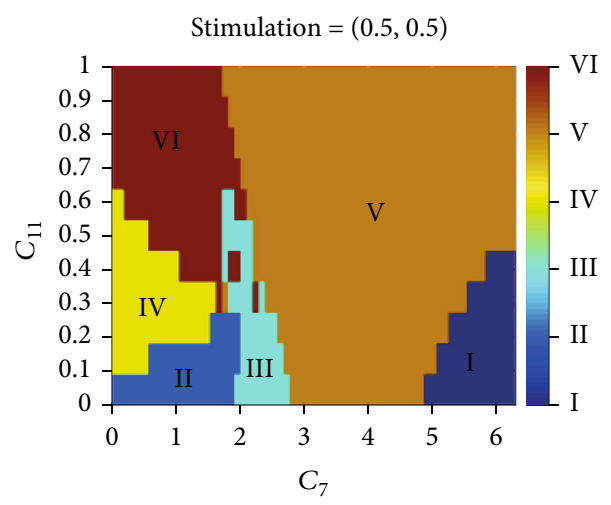

(c)

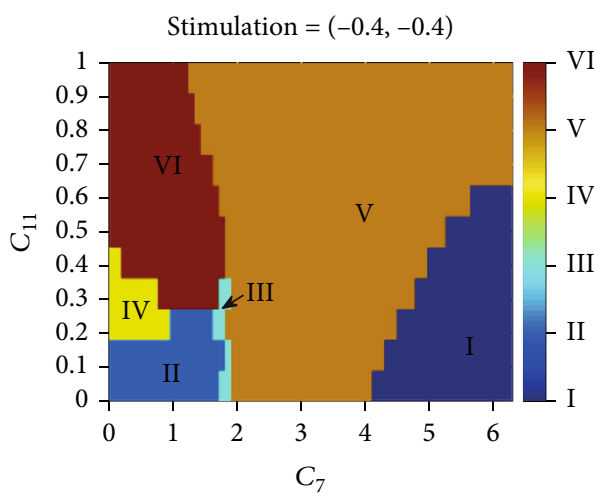

(e)

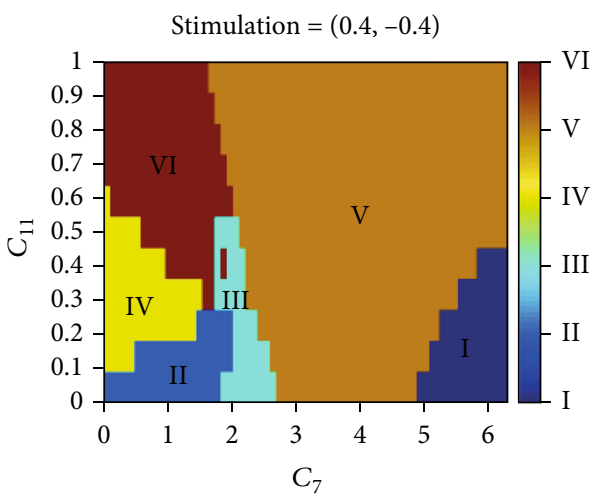

(b)

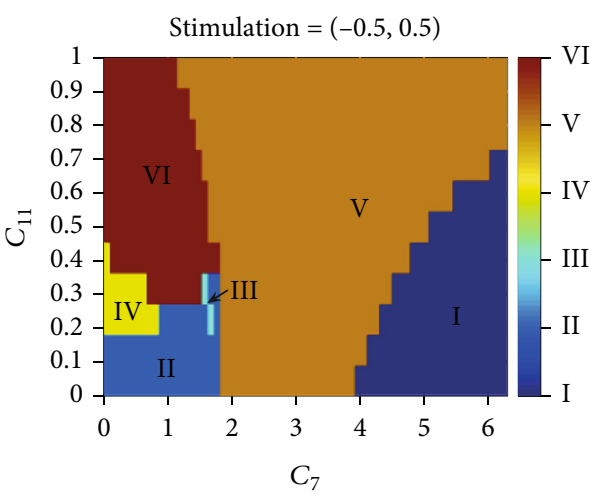

(d)

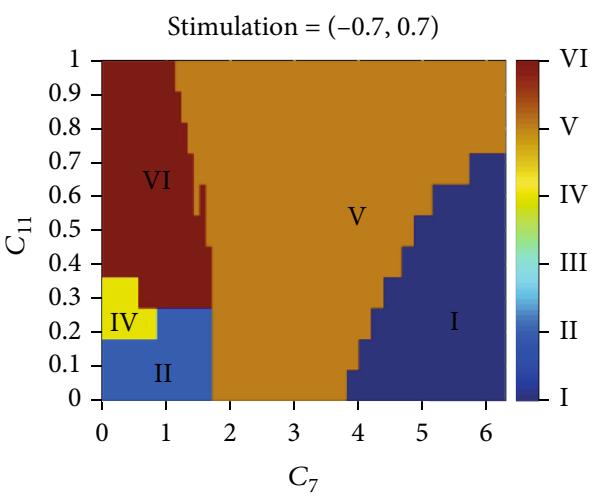

(f)

FIGURE 12: State types after applying CRS with different stimulus intensities on RE and TC, where I (high saturated state), II (low saturated state), III (SWD), IV (tonic), V (low-frequency clonic), and VI (high-frequency clonic) are six different firing states. (a-f) Is the twodimensional parametric plane $\left(C_{7}, C_{11}\right)$ without CRS. These typical two-dimensional plane diagrams show that SWD can be controlled well by CRS. And $(-0.7,0.7)$ is the most effective strategy for absence epilepsy.

And the other is current consumption, which can be expressed as follows:

$$
I_{\text {current }}=\frac{1}{\sqrt{N}} \bullet\left\|I_{\text {stim }}(t)\right\|_{2} \text {, }
$$

where $I_{\text {stim }}(t),\|\bullet\|_{2}$, and $N$ represent the stimulus intensity on (RE, TC), two norms of $I_{\text {stim }}(t)$, and total time steps, respectively.
Here, we set the frequency and pulse width of stimulus which are $100 \mathrm{~Hz}$ and $0.001 \mathrm{~s}$. Since the accumulation of electric charge will cause side effects to patients, we firstly explore the strategies of applying the anodic pulse stimulus to RE and cathodic pulse stimulus to TC. For example, SWD region is reduced by about $13 \%$ when the stimulus intensity is $(0.4$, -0.4) (see Figure 12(b)). And SWD area will decrease as the stimulus amplitude increases (see Figure 13(a)). However, if we apply negative pulse stimulus to RE and positive pulse stimulus to TC, the control effect will be greatly improved, even up to $100 \%$ as the stimulus intensity is $(-0.7,0.7)$ (see 


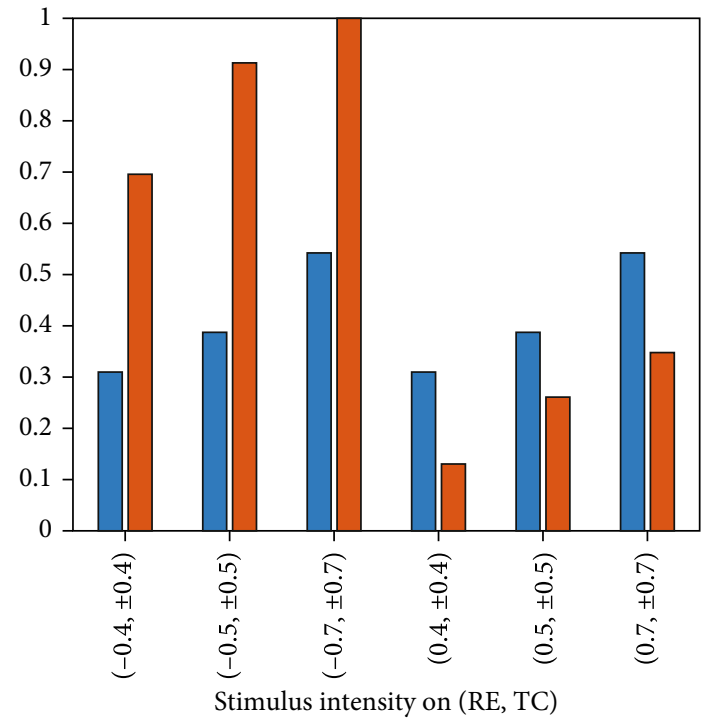

Current consumption

Stimulation effect on SWD

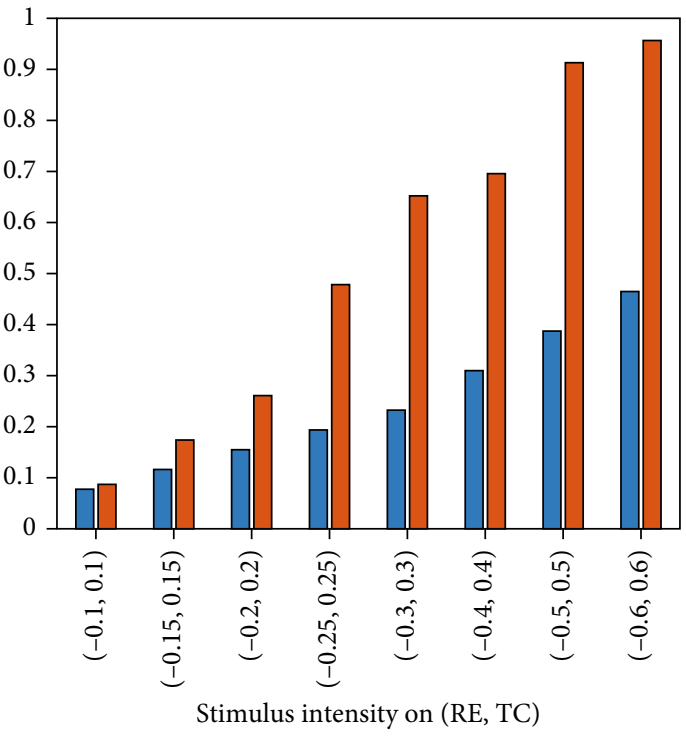

Current consumption

Stimulation effect on SWD

(b)

FIGURE 13: Stimulation effect on SWD and current consumption under different CRS stimulus intensities. The stimulus applied to RE can be positive or negative (a), while in (b), it is only negative. It can be seen from the bar graph that the greater the stimulation intensity, the best the control effect, although more and more energy is consumed.

Figure 12(f)). Then, we further verify that the greater the stimulus amplitude, the better the control effect (see Figure 13(b)). We also exhibit the control percentage $\eta$ when the same nature of the stimulus is applied to our targets (see Figures 12(c) and 12(e)). Although SWD also can be controlled in this way, the accumulation of charge can cause some harm to the patient. Hence, we think that maybe $(-0.7,0.7)$ is a more effective strategy for absence epilepsy.

\section{Discussions and Conclusions}

It is demonstrated that there exists a basic disinhibition circuit among inhibitory neurons with different time scales [45]. And considering the fact that feedforward inhibition from TC has even more stronger functions on the cortex than the feedforward excitation in some cortical areas [46], we modify the classical Taylor neural field model to provide more theoretical foundation for the absence epilepsy treatment.

As described in many experimental results, astrocytes are involved in epilepsy [22-26]. The findings of the present study also highlight the potential of astrocyte as a useful complementary therapy for absence epilepsy. Rich transition dynamics can be induced by astrocyte dysfunction. If astrocytes are normal, the system will show normal low saturated state. However, when the function of astrocytes is inhibited or overexcited, pathological brain waves such as SWD, tonic, and clonic will appear.

Although single-pulse stimulation has improved in the last few years, the mechanisms are not entirely clear. At the same time, considering the important role of RE, we specifically show that when the system is in different initial states, the system can exhibit attractive dynamic transition with the increase of stimulus intensity after applying stimulation to RE. We find that single-pulse stimulation can not only suppress but also induce pathological firing patterns. Then, we applied DBS, a more clinically meaningful stimulation strategy to RE. The results suggest that DBS can control absence epilepsy by regulating the amplitude and pulse width of stimulation. Furthermore, we study another safer and more popular stimulation strategy, which is 3:2 CRS with different stimulation intensities. Our results show that the control effect on SWD will be greatly improved as the stimulus amplitude increases. However, results obtained through theoretical models still need to be compared with medical experiments and clinical practice in our future works.

It should be noted that we only studied the effect of a specific function of astrocytes on the pathological structure of neural networks, and the multiple-scale network models of the interneuron, pyramidal cell, and the astrocyte should be developed in our next work. In addition, more and more scholars pay attention to dynamic phenomena generated by random factors [60-62]. We hope to conduct a comprehensive study in this area.

\section{Data Availability}

The data used to support the findings of this study are included within the article. 


\section{Conflicts of Interest}

The authors declare that there is no conflict of interest regarding the publication of this paper.

\section{Acknowledgments}

The research was supported by the Natural Science Foundation of China (Nos. 11872304, 12072265 and 11972292) and Postdoctoral Research Foundation of China (No. 2017M623233).

\section{References}

[1] Z. Barad, D. R. Grattan, and B. Leitch, "NMDA receptor expression in the thalamus of the stargazer model of absence epilepsy," Scientific Reports, vol. 7, no. 1, article 42926, 2017.

[2] A. Salek-Haddadi, L. Lemieux, M. Merschhemke, K. J. Friston, J. S. Duncan, and D. R. Fish, "Functional magnetic resonance imaging of human absence seizures," Annals of Neurology, vol. 53, no. 5, pp. 663-667, 2003.

[3] L. G. Sadleir, K. Farrell, S. Smith, M. B. Connolly, and I. E. Scheffer, "Electroclinical features of absence seizures in childhood absence epilepsy," Neurology, vol. 67, no. 3, pp. 413418, 2006.

[4] G. L. Holmes, M. McKeever, and M. Adamson, "Absence seizures in children: clinical and electroencephalographic features," Annals of Neurology, vol. 21, no. 3, pp. 268-273, 1987.

[5] S. Pilge, "Epileptiform electroencephalographic activity and generalized tonic-clonic seizures: case report," Reactions Weekly, vol. 1538, pp. 214-214, 2015.

[6] J. Gao, S.-T. Feng, B. Wu et al., "Microstructural brain abnormalities of children of idiopathic generalized epilepsy with generalized tonic-clonic seizure: a voxel-based diffusional kurtosis imaging study," Journal of Magnetic Resonance Imaging, vol. 41, no. 4, pp. 1088-1095, 2015.

[7] J. Dobesberger, A. J. Ristić, G. Walser et al., "Duration of focal complex, secondarily generalized tonic-clonic, and primarily generalized tonic-clonic seizures-a video-EEG analysis," Epilepsy \& Behavior, vol. 49, pp. 111-117, 2015.

[8] J. Engel, T. A. Pedley, and J. Aicardi, Epilepsy: A Comprehensive Textbook, Lippincott Williams \& Wilkins, 2008.

[9] G.-J. Ji, Z. Zhang, Q. Xu, Y.-F. Zang, W. Liao, and G. Lu, "Generalized tonic-clonic seizures: aberrant interhemispheric functional and anatomical connectivity," Radiology, vol. 271, no. 3, pp. 839-847, 2014.

[10] M. Curtisde and V. Gnatkovsky, "Reevaluating the mechanisms of focal ictogenesis: the role of low-voltage fast activity," Epilepsia, vol. 50, no. 12, pp. 2514-2525, 2009.

[11] M. A. Kramer and S. S. Cash, "Epilepsy as a disorder of cortical network organization," The Neuroscientist, vol. 18, no. 4, pp. 360-372, 2012.

[12] A. Depaulis, O. David, and S. Charpier, "The genetic absence epilepsy rat from Strasbourg as a model to decipher the neuronal and network mechanisms of generalized idiopathic epilepsies," Journal of Neuroscience Methods, vol. 260, pp. 159-174, 2016.

[13] P.-O. Polack, I. Guillemain, E. Hu, C. Deransart, A. Depaulis, and S. Charpier, "Deep layer somatosensory cortical neurons initiate spike-and-wave discharges in a genetic model of absence seizures," The Journal of Neuroscience, vol. 27, no. 24, pp. 6590-6599, 2007.

[14] M. Steriade and D. Contreras, "Spike-wave complexes and fast components of cortically generated seizures. I. Role of neocortex and thalamus," Journal of Neurophysiology, vol. 80, no. 3, pp. 1439-1455, 1998.

[15] T. Bal, D. Debay, and A. Destexhe, "Cortical feedback controls the frequency and synchrony of oscillations in the visual thalamus," The Journal of Neuroscience, vol. 20, no. 19, pp. 74787488, 2000.

[16] A. M. L. Coenen and E. L. J. M. van Luijtelaar, "Genetic animal models for absence epilepsy: a review of the WAG/Rij strain of rats," Behavior Genetics, vol. 33, no. 6, pp. 635-655, 2003.

[17] I. Timofeev and M. Steriade, "Neocortical seizures: initiation, development and cessation," Neuroscience, vol. 123, no. 2, pp. 299-336, 2004.

[18] A. Araque, V. Parpura, R. P. Sanzgiri, and P. G. Haydon, "Tripartite synapses: glia, the unacknowledged partner," Trends in Neurosciences, vol. 22, no. 5, pp. 208-215, 1999.

[19] H. R. Parri, T. M. Gould, and V. Crunelli, "Spontaneous astrocytic $\mathrm{Ca}^{2+}$ oscillations in situ drive NMDAR-mediated neuronal excitation," Nature Neuroscience, vol. 4, no. 8, pp. 803-812, 2001.

[20] K. W. Young, M. S. Nash, R. A. J. Challiss, and S. R. Nahorski, "Role of $\mathrm{Ca} 2+$ feedback on single cell inositol 1,4,5-trisphosphate oscillations mediated by G-protein-coupled receptors," Journal of Biological Chemistry, vol. 278, no. 23, pp. 2075320760, 2003.

[21] T. Fellin and G. Carmignoto, "Neurone-to-astrocyte signalling in the brain represents a distinct multifunctional unit," The Journal of Physiology, vol. 559, no. 1, pp. 3-15, 2004.

[22] C. Steinhäuser and G. Seifert, "Astrocyte dysfunction in temporal lobe epilepsy," Epilepsia, vol. 51, p. 54, 2010.

[23] G. Seifert, G. Carmignoto, and C. Steinhäuser, "Astrocyte dysfunction in epilepsy," Brain Research Reviews, vol. 63, no. 1-2, pp. 212-221, 2010.

[24] O. K. Steinlein, "Calcium signaling and epilepsy," Cell \& Tissue Research, vol. 357, no. 2, pp. 385-393, 2014.

[25] D. A. Coulter and C. Steinhäuser, "Role of astrocytes in epilepsy," Cold Spring Harbor Perspectives in Medicine, vol. 5, no. 3, pp. 649-671, 2015.

[26] V. Crunelli, G. Carmignoto, and C. Steinhäuser, "Novel astrocyte targets," The Neuroscientist, vol. 21, no. 1, pp. 62-83, 2015.

[27] G.-F. Tian, H. Azmi, T. Takano et al., "An astrocytic basis of epilepsy,” Nature Medicine, vol. 11, no. 9, pp. 973-981, 2005.

[28] T. Fellin, O. Pascual, S. Gobbo, T. Pozzan, P. G. Haydon, and G. Carmignoto, "Neuronal synchrony mediated by astrocytic glutamate through activation of extrasynaptic NMDA receptors," Neuron, vol. 43, no. 5, pp. 729-743, 2004.

[29] T. Fellin and P. G. Haydon, "Do astrocytes contribute to excitation underlying seizures?," Trends in Molecular Medicine, vol. 11, no. 12, pp. 530-533, 2005.

[30] T. Fellin, M. Gomez-Gonzalo, S. Gobbo, G. Carmignoto, and P. G. Haydon, "Astrocytic glutamate is not necessary for the generation of epileptiform neuronal activity in hippocampal slices," The Journal of Neuroscience, vol. 26, no. 36, pp. 9312 9322, 2006.

[31] J. Li, J. Tang, J. Ma, M. Du, R. Wang, and Y. Wu, "Dynamic transition of neuronal firing induced by abnormal astrocytic glutamate oscillation," Scientific Reports, vol. 6, no. 1, article 32343, 2016. 
[32] J. Li, R. Wang, M. Du, J. Tang, and Y. Wu, "Dynamic transition on the seizure-like neuronal activity by astrocytic calcium channel block," Chaos Solitons \& Fractals, vol. 91, pp. 702708, 2016.

[33] J. J. Li, Y. Xie, Y. G. Yu, M. M. du, R. Wang, and Y. Wu, “A neglected GABAergic astrocyte: calcium dynamics and involvement in seizure activity," Science China-Technological Sciences, vol. 60, no. 7, pp. 1003-1010, 2017.

[34] M. H. Myers and R. Kozma, "Mesoscopic neuron population modeling of normal/epileptic brain dynamics," Cognitive Neurodynamics, vol. 12 , no. 2, pp. 211-223, 2018.

[35] M. Chen, D. Guo, T. Wang et al., "Bidirectional control of absence seizures by the basal ganglia: a computational evidence," PLoS Computational Biology, vol. 10, no. 3, article e1003495, 2014.

[36] B. Hu, D. Guo, and Q. Wang, "Control of absence seizures induced by the pathways connected to SRN in corticothalamic system," Cognitive Neurodynamics, vol. 9, no. 3, pp. 279-289, 2015.

[37] P. N. Taylor, Y. Wang, M. Goodfellow et al., "A computational study of stimulus driven epileptic seizure abatement," PLoS One, vol. 9, no. 12, p. e114316, 2014.

[38] P. N. Taylor, J. Thomas, N. Sinha et al., "Optimal control based seizure abatement using patient derived connectivity," Frontiers in Neuroscience, vol. 9, p. 202, 2015.

[39] D. Fan, L. Duan, Q. Wang, and G. Luan, "Combined effects of feedforward inhibition and excitation in thalamocortical circuit on the transitions of epileptic seizures," Frontiers in Computational Neuroscience, vol. 11, p. 59, 2017.

[40] S. Liu, Q. Wang, and D. Fan, "Disinhibition-induced delayed onset of epileptic spike-wave discharges in a five variable model of cortex and thalamus," Frontiers in Computational Neuroscience, vol. 10, p. 28, 2016.

[41] P. Kwan and M. J. Brodie, "Early identification of refractory epilepsy," The New England Journal of Medicine, vol. 342, no. 5, pp. 314-319, 2000.

[42] J. Engel, S. Wiebe, J. French et al., "Practice parameter: temporal lobe and localized neocortical resections for epilepsy," Epilepsia, vol. 44, no. 6, pp. 741-751, 2003.

[43] Y. Ge, Y. Cao, G. Yi et al., "Robust closed-loop control of spikeand-wave discharges in a thalamocortical computational model of absence epilepsy," Scientific Reports, vol. 9, no. 1, p. 9093, 2019.

[44] Z. Wang and Q. Wang, "Stimulation strategies for absence seizures: targeted therapy of the focus in coupled thalamocortical model," Nonlinear Dynamics, vol. 96, no. 2, pp. 1649-1663, 2019.

[45] H.-J. Pi, B. Hangya, D. Kvitsiani, J. I. Sanders, Z. J. Huang, and A. Kepecs, "Cortical interneurons that specialize in disinhibitory control," Nature, vol. 503, no. 7477, pp. 521-524, 2013.

[46] B. Beverlin, J. Kakalios, D. Nykamp, and T. I. Netoff, "Dynamical changes in neurons during seizures determine tonic to clonic shift," Journal of Computational Neuroscience, vol. 33, no. 1, pp. 41-51, 2012.

[47] A. Bacci, C. Verderio, E. Pravettoni, and M. Matteoli, "The role of glial cells in synaptic function," Philosophical Transactions of the Royal Society B, vol. 354, no. 1381, pp. 403-409, 1999.

[48] S. K. Suchak, N. V. Baloyianni, M. S. Perkinton, R. J. Williams, B. S. Meldrum, and M. Rattray, "The 'glial' glutamate transporter, EAAT2 (Glt-1) accounts for high affinity glutamate uptake into adult rodent nerve endings," Journal of Neurochemistry, vol. 84, no. 3, pp. 522-532, 2003.

[49] Z. Liu, M. Vergnes, A. Depaulis, and C. Marescaux, "Evidence for a critical role of GABAergic transmission within the thalamus in the genesis and control of absence seizures in the rat," Brain Research, vol. 545, no. 1-2, pp. 1-7, 1991.

[50] J. R. Tenney, T. Q. Duong, J. A. King, R. Ludwig, and C. F. Ferris, "Corticothalamic modulation during absence seizures in rats: a functional MRI assessment," Epilepsia, vol. 44, no. 9, pp. 1133-1140, 2003.

[51] G. L. Holmes and Y. Ben-Ari, "The neurobiology and consequences of epilepsy in the developing brain," Pediatric Research, vol. 49, no. 3, pp. 320-325, 2001.

[52] Y. J. John, B. Zikopoulos, D. Bullock, and H. Barbas, "The emotional gatekeeper: a computational model of attentional selection and suppression through the pathway from the amygdala to the inhibitory thalamic reticular nucleus," PLOS Computational Biology, vol. 12, no. 2, p. e1004722, 2016.

[53] A. M. Willis, B. J. Slater, E. D. Gribkova, and D. A. Llano, "Open-loop organization of thalamic reticular nucleus and dorsal thalamus: a computational model," Journal of Neurophysiology, vol. 114, no. 4, pp. 2353-2367, 2015.

[54] J. B. Eells, R. W. Clough, R. A. Browning, and P. C. Jobe, "Comparative Fos immunoreactivity in the brain after forebrain, brainstem, or combined seizures induced by electroshock, pentylenetetrazol, focally induced and audiogenic seizures in rats," Neuroscience, vol. 123, no. 1, pp. 279-292, 2004.

[55] C. Mayville, T. Fakhoury, and B. Abou-Khalil, "Absence seizures with evolution into generalized tonic-clonic activity: clinical and EEG features," Epilepsia, vol. 41, no. 4, pp. 391394, 2000.

[56] P. N. Taylor, G. Baier, S. S. Cash, J. Dauwels, J.-J. Slotine, and Y. Wang, "A model of stimulus induced epileptic spike-wave discharges," in 2013 IEEE Symposium on Computational Intelligence, Cognitive Algorithms, Mind, and Brain (CCMB), pp. 53-59, Singapore, 2013.

[57] T. Loddenkemper, A. Pan, S. Neme et al., "Deep brain stimulation in epilepsy," Journal of Clinical Neurophysiology, vol. 18, no. 6, pp. 514-532, 2001.

[58] P. Boon, K. Vonck, V. de Herdt et al., "Deep brain stimulation in patients with refractory temporal lobe epilepsy," Epilepsia, vol. 48 , no. 8, pp. 1551-1560, 2007.

[59] R. S. Fisher and A. L. Velasco, "Electrical brain stimulation for epilepsy," Nature Reviews Neurology, vol. 10, no. 5, pp. 261270, 2014.

[60] Y. Yao and J. Ma, "Weak periodic signal detection by sine-wiener-noise-induced resonance in the FitzHugh-Nagumo neuron," Cognitive Neurodynamics, vol. 12, no. 3, pp. 343-349, 2018.

[61] O. Nakamura and K. Tateno, "Random pulse induced synchronization and resonance in uncoupled non-identical neuron models," Cognitive Neurodynamics, vol. 13, no. 3, pp. 303-312, 2019.

[62] N. Sinha, Y. Wang, J. Dauwels et al., "Computer modelling of connectivity change suggests epileptogenesis mechanisms in idiopathic generalised epilepsy," NeuroImage: Clinical, vol. 21, article 101655, 2019. 